\title{
High-resolution SMA imaging of bright submillimetre sources from the SCUBA-2 Cosmology Legacy Survey
}

\author{
Ryley Hill, ${ }^{1}$ Scott C. Chapman, ${ }^{2}$ Douglas Scott, ${ }^{1}$ Glen Petitpas, ${ }^{3}$ Ian Smail,${ }^{4}$
} Edward L. Chapin, ${ }^{5}$ Mark A. Gurwell, ${ }^{3}$ Ryan Perry, ${ }^{2}$ Andrew W. Blain, ${ }^{6}$ Malcolm N. Bremer, ${ }^{7}$ Chian-Chou Chen, ${ }^{4,8}$ James S. Dunlop, ${ }^{9}$ Duncan Farrah, ${ }^{10}$

Giovanni G. Fazio, ${ }^{3}$ James E. Geach, ${ }^{11}$ Paul Howson, ${ }^{2}$ R. J. Ivison, ${ }^{8,9}$ Kevin Lacaille, ${ }^{2,12}$ Michał J. Michałowski, ${ }^{13}$ James M. Simpson, ${ }^{9,14}$ A. M. Swinbank, ${ }^{4}$ Paul P. van der Werf, ${ }^{15}$ David J. Wilner ${ }^{3}$

${ }^{1}$ Department of Physics and Astronomy, University of British Columbia, 6225 Agricultural Road, Vancouver, V6T 1Z1, Canada

${ }^{2}$ Department of Physics and Atmospheric Science, Dalhousie University, 6310 Coburg Road, Halifax, B3H 4R2, Canada

${ }^{3}$ Harvard-Smithsonian Center for Astrophysics, 60 Garden Street, Cambridge, MA 02138, USA

${ }^{4}$ Centre for Extragalactic Astronomy, Department of Physics, Durham University, South Road, Durham, DH1 3LE, UK

${ }^{5}$ Herzberg Astronomy and Astrophysics, National Research Council Canada, 5071 West Saanich Road, Victoria, V9E 2E7, Canada

${ }^{6}$ Department of Physics and Astronomy, University of Leicester, University Road, Leicester, LE1 7RH, UK

${ }^{7}$ H.H. Wills Physics Laboratory, University of Bristol, Tyndall Avenue, Bristol, BS8 1TL, UK

${ }^{8}$ European Southern Observatory, Karl-Schwarzschild-Straße 2, Garching, 85748, Germany

${ }^{9}$ Institute for Astronomy, University of Edinburgh, Royal Observatory, Edinburgh, EH9 $3 H J$, UK

${ }^{10}$ Department of Physics MC 0435, Virginia Polytechnic Institute and State University, 850 West Campus Drive, Blacksburg, VA 24061, USA

${ }^{11}$ Centre for Astrophysics Research, School of Physics, Astronomy and Mathematics, University of Hertfordshire, Roehyde Way, Hatfield, AL10 $9 A B, U K$

${ }^{12}$ Department of Physics and Astronomy, McMaster University, 1280 Main Street West, Hamilton, L8S 4M1, Canada

${ }^{13}$ Astronomical Observatory Institute, Faculty of Physics, Adam Mickiewicz University, ul. Stoneczna 36, 60-286 Poznań, Poland

${ }^{14}$ Academia Sinica Institute of Astronomy and Astrophysics, No. 1, Sec. 4, Roosevelt Road, Taipei 10617, Taiwan

${ }^{15}$ Leiden Observatory, Leiden University, P.O. box 9513, Leiden, NL-2300 RA, the Netherlands

\begin{abstract}
We have used the Submillimeter Array at $860 \mu \mathrm{m}$ to observe the brightest SCUBA-2 sources in $4 \mathrm{deg}^{2}$ of the Cosmology Legacy Survey. We have targeted 75 of the brightest single-dish SCUBA-2 $850 \mu \mathrm{m}$ sources down to $S_{850} \approx 8 \mathrm{mJy}$, achieving an average synthesized beam of 2.4 arcsec and an average rms of $\sigma_{860}=1.5 \mathrm{mJy}$ in our primary beamcorrected maps. We searched our maps for $4 \sigma$ peaks, corresponding to $S_{860} \gtrsim 6 \mathrm{mJy}$ sources, and detected 59 single galaxies and three pairs of galaxies. We include in our study 28 archival observations, bringing our sample size to 103 bright singledish submillimetre sources with interferometric follow-up. We compute the cumulative and differential number counts of our sample, finding them to overlap with previous single-dish survey number counts within the uncertainties, although our cumulative number count is systematically lower than the parent SCUBA-2 cumulative number count by $24 \pm 6$ per cent between 11 and $15 \mathrm{mJy}$. We estimate the probability that a $\gtrsim 10$ mJy single-dish submillimetre source resolves into two or more galaxies with similar flux densities, causing a significant change in the number counts, to be about 15 per cent. Assuming the remaining 85 per cent of the targets are ultra-luminous starburst galaxies between $z=2-3$, we find a likely volume density of $\gtrsim 400 \mathrm{M}_{\odot} \mathrm{yr}^{-1}$ sources to be $\sim 3_{-0.6}^{+0.7} \times 10^{-7} \mathrm{Mpc}^{-3}$. We show that the descendants of these galaxies could be $\gtrsim 4 \times 10^{11} \mathrm{M}_{\odot}$ local quiescent galaxies, and that about 10 per cent of their total stellar mass would have formed during these short bursts of star-formation.
\end{abstract}

Key words: submillimetre: galaxies - galaxies: statistics - galaxies: starburst 


\section{INTRODUCTION}

The emergence of submillimetre (submm) astronomy has led to the discovery of a cosmologically important population of submm galaxies (SMGs), which appear to be among the earliest and most actively star-forming galaxies in the Universe, often reaching luminosities of a few times $10^{13} \mathrm{~L}_{\odot}$ and star-formation rates (SFRs) greater than a few hundred $\mathrm{M}_{\odot} \mathrm{yr}^{-1}$ (e.g., Blain et al. 2002; Magnelli et al. 2012; Swinbank et al. 2014; MacKenzie et al. 2017; Michałowski et al. 2017) and above (e.g. HFLS3, see Riechers et al. 2013) around redshifts 2-3 (e.g., Chapman et al. 2005; Simpson et al. 2014, 2017). The Submillimeter Common User Bolometer Array (SCUBA; Holland et al. 1999), mounted on the 15-m James Clerk Maxwell Telescope (JCMT), was the first multi-pixel instrument to detect this population of highredshift SMGs (e.g. Smail et al. 1997; Barger et al. 1998; Hughes et al. 1998). This motivated the development of more sensitive detectors such as the second generation SCUBA2 (Holland et al. 2013), the Large Apex BOlometer CAmera (LABOCA; Siringo et al. 2009), and the AZtronomical Thermal Emission Camera (AzTEC; Wilson et al. 2008), as well as the Balloon-borne Large Aperture Submillimeter Telescope (BLAST; Pascale et al. 2008) and the space-based Spectral and Photometric Imaging REceiver (SPIRE; Griffin et al. 2010) on board the Herschel satellite, all of which have been used to further investigate SMGs.

While single dish observations of SMGs were able to greatly increase our knowledge about the evolution of star formation in the Universe (e.g., Blain et al. 1999; Magnelli et al. 2013; Gruppioni et al. 2013; Swinbank et al. 2014; Koprowski et al. 2017), their connection with today's galaxies remains unclear, although evidence is mounting that they are progenitors of massive elliptical galaxies (e.g., Lilly et al. 1999; Scott et al. 2002; Genzel et al. 2003; Swinbank et al. 2006; Toft et al. 2014; Simpson et al. 2014; Koprowski et al. 2014; van Dokkum et al. 2015; Koprowski et al. 2016; Michałowski et al. 2017; Simpson et al. 2017). There is also debate about whether or not mergers are important for SMGs. Many simulations require mergers to achieve the observed massive SFRs (e.g., Narayanan et al. 2015) while others do not (e.g., Davé et al. 2010), and on the other hand, observations of physically associated pairs of SMGs with disturbed gas morphologies indicate that mergers are present (e.g., Tacconi et al. 2008; Engel et al. 2010; Chen et al. 2015), while ultra-luminous SMGs have been seen that lack evidence of multiplicity and fit on the high-mass end of the 'main sequence' of star-forming galaxies (e.g., Targett et al. 2013; Michałowski et al. 2017). Progress is impeded by the sub-optimal angular resolution offered by single dish telescopes at submm wavelengths, which typically ranges between 10 and 30 arcseconds. At these scales, source blending becomes a significant problem, and optical/near infrared (NIR) counterparts cannot be easily identified.

This problem was first tackled by exploiting the high spatial resolution available to interferometers operating in the radio waveband, where synchrotron emission linked to supernovae is very closely correlated with far-infrared emission from dust (e.g., Condon 1992; Yun et al. 2001; Ivison et al. 2010; Magnelli et al. 2015) - dust thought to be created following those same supernova events (e.g. Indebetouw et al. 2014) and heated by young, massive stars. Radio stud- ies of SMGs were typically able to determine positions to subarcsec accuracy, and thus localize multiwavelength counterpart galaxies using probabilistic arguments (e.g., Chapman et al. 2001; Ivison et al. 2002; Chapman et al. 2002, 2003; Bertoldi et al. 2007; Biggs et al. 2011), which greatly improved our understanding of their redshift distribution (Smail et al. 2000; Chapman et al. 2003, 2005; Dannerbauer et al. 2004; Smolčić et al. 2012a) and physical characteristics (Ivison et al. 1998, 2000; Smail et al. 2000; Chapman et al. 2004; Borys et al. 2004). In particular, Ivison et al. (2007) showed that a significantly larger fraction of SMGs contained multiple radio counterparts than would be expected by chance, suggesting therefore that they could comprise groups of physically associated galaxies.

However, more accurately pinpointing the submm emission directly - the only way to be fully sure that the associated positions and optical/IR counterparts are bone fidewas not possible until the leap in continuum sensitivity provided by new submm interferometers and wide-bandwidth correlators, such as those available at the Plateau de Bure Interferometer (PdBI; Guilloteau et al. 1992), the Submillimeter Array (SMA; Ho et al. 2004) and, most recently, the Atacama Large Millimeter/submillimeter Array (ALMA; Wootten \& Thompson 2009). These have greatly aided the localisation of counterparts and the further characterization of SMGs. These facilities were able to confirm that many SMGs exhibit multiplicity (e.g., Iono et al. 2006; Younger et al. 2007, 2009; Wang et al. 2011; Smolčić et al. 2012b; Hodge et al. 2013; Simpson et al. 2015; Miettinen et al. 2015, Stach et al. in prep.), where one bright single-dish submm source resolves into two or three individual SMGs.

Large single-dish submm surveys (e.g., Scott et al. 2002; Greve et al. 2004; Wang et al. 2004; Coppin et al. 2006; Bertoldi et al. 2007; Weiß et al. 2009; Oliver et al. 2010; Valiante et al. 2016; Geach et al. 2017), followed up by interferometers, have been important for addressing the issue of multiplicity as they provide substantial catalogues of bright single-dish sources across continuous patches of sky that interferometers can follow-up. These types of studies are able to distinguish between intrinsically bright galaxies and systems comprising of two or more galaxies with similar flux densities that would lead to a significant overestimation from single-dish measurements. For example, Barger et al. (2012) used the SMA to observe $16 S_{850}>3$ mJy sources detected with SCUBA-2 in the Great Observatories Origins Deep Survey-North field (GOODS-N; Wang et al. 2004), finding that three resolved into multiple SMGs. Similarly, Smolčić et al. (2012b) used the PdBI at $1.3 \mathrm{~mm}$ to target $28 S_{870}>5 \mathrm{mJy}$ sources detected by LABOCA at $870 \mu \mathrm{m}$ in the COSMOS field, and found that six of them resolved into more than one SMG. A larger LABOCA $0.25 \mathrm{deg}^{2}$ survey of the Extended Chandra Deep Field South (LESS; Weiß et al. 2009) was followed up with ALMA by Hodge et al. (2013), who observed 126 sources $S_{870}>3.5 \mathrm{mJy}$ and found that 24 out of the 69 most robust observations showed multiple SMGs. More recently, Simpson et al. (2015) used ALMA at $870 \mu \mathrm{m}$ to follow-up 30 of the brightest $\left(S_{850}>5 \mathrm{mJy}\right)$ sources detected in the UKIDSS-UDS field at $850 \mu \mathrm{m}$, mapped by SCUBA-2 as part of the Cosmology Legacy Survey (CLS; Geach et al. 2017), and found that 18 sources break up into more than two SMGs. While these types of surveys have begun to reach statistically significant numbers of sam- 
ples, they nonetheless lack large numbers of the brightest single dish detected sources; for example, the LESS survey contained 20 sources with $S_{850}>8 \mathrm{mJy}$ and six sources with $S_{850}>10 \mathrm{mJy}$, and the observations from Simpson et al. (2015) contained 13 sources with $S_{850}>8$ and seven sources with $S_{850}>10 \mathrm{mJy}$ when considering the final CLS catalogue.

To date, the largest submm survey of the extragalactic sky is the complete CLS, encompassing $5 \mathrm{deg}^{2}$ of the sky over seven cosmological fields: UKIDSS-UDS, COSMOS, AkariNEP, Extended Groth Strip, Lockman Hole North, SSA22 and GOODS-North. The CLS detected over 2800 submm sources above $3.5 \sigma$, where 114 of them had $S_{850}>8 \mathrm{mJy}$ and 46 of them had $S_{850}>10 \mathrm{mJy}$. This survey is therefore wellsuited to study the properties of multiplicity in the brightest SMGs known to exist.

In this paper we present results from the largest yet interferometric follow-up programme of the brightest submm galaxies, selected from 80 per cent of the available area in the CLS survey. We have imaged 75 SCUBA-2 sources with $S_{850} \geq 8 \mathrm{mJy}$ with 2.4 arcsec resolution using the SMA in order to measure the importance of multiplicity in this bright population of SMGs. In Section 2 we describe our target selection, data reduction and source extraction procedure, in Section 3 we correct our flux density measurements for flux boosting and compare our data to the CLS catalogue to asses the reliability of our sample, and in Section 4 we examine the completeness of our sample, present number counts, and discuss the effects of multiplicity on the population of bright SMGs seen in our data. We give our conclusions in Section 5

\section{OBSERVATIONS AND DATA REDUCTION}

\subsection{Target selection}

In our observing programme we used the SMA in the compact configuration at $860 \mu \mathrm{m}$ to investigate bright sources in five out of the seven wide $850-\mu \mathrm{m}$ CLS fields, namely UKIDSS-UDS, COSMOS, the Extended Groth Strip, the Lockman Hole North, and SSA22 (hereafter the UDS, COSMOS, EGS, LHN and SSA22 fields, respectively). Combined, these fields make up about 80 per cent of the full CLS at $4 \mathrm{deg}^{2}$, and contain 98 sources with $S_{850}>8 \mathrm{mJy}$ and 39 sources with $S_{850}>10 \mathrm{mJy}$. Our initial aim was to target and resolve all sources down to $\approx 8 \mathrm{mJy}$ and to determine the effects of multiplicity on the bright end of the submm number count with good statistical significance. At the time these observations were first proposed, the CLS had not yet been completed, being at that point shallower than the final maps published in Geach et al. (2017). This led to several cases where either a proposed SCUBA-2 target ended up fainter than expected, or an originally faint SCUBA2 source ended up being brighter than $10 \mathrm{mJy}$ at $850 \mu \mathrm{m}$. When selecting targets we only considered the measured (uncorrected) SCUBA-2 flux densities, which are believed to be boosted by positive noise and faint background galaxies that on average add a positive bias to the flux densities and are statistically corrected for in the final CLS catalogue in Geach et al. (2017). This effect resulted in more examples of apparently bright SCUBA-2 sources ending up being fainter in the final list.

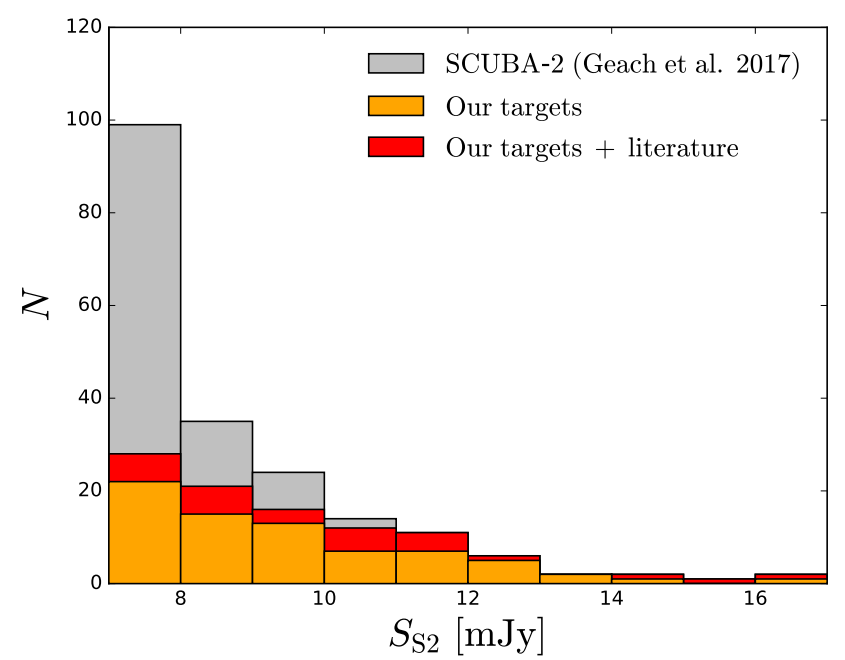

Figure 1. Histogram showing the deboosted flux density distribution of the parent SCUBA-2 CLS survey from Geach et al. (2017), our 75 targets, and our full catalogue including these 75 targets and 28 archival sources from Simpson et al. (2015), Younger et al. (2007), Younger et al. (2009) and Ikarashi et al. (2011), which are included in our counts analysis. Our sample is a nearly complete selection of single-dish $850-\mu \mathrm{m}$ SCUBA-2 sources with flux densities brighter than $10 \mathrm{mJy}$.

There are several submm interferometric data sets from the SMA and ALMA in the literature that we did not reobserve in our programme. In particular, Simpson et al. (2015) carried out a follow-up campaign of 30 bright CLS sources in the UDS field with ALMA at $870 \mu \mathrm{m}$, and Younger et al. $(2007,2009)$ selected the highest significance sources in an AzTEC $1.1 \mathrm{~mm}$ survey of the COSMOS field (Scott et al. 2008), many of which are also bright at $850 \mu \mathrm{m}$ in the SCUBA-2 map, for follow-up with the SMA at $890 \mu \mathrm{m}$. Additionally, there is a single strong gravitational lens in the UDS field, dubbed 'Orochi', reaching an 850- $\mu \mathrm{m}$ flux density of $52.7 \mathrm{mJy}$ in the SCUBA-2 map; this source was followed up by Ikarashi et al. (2011) with the SMA at $860 \mu \mathrm{m}$ in part of a detailed multiwavelength study. We have included 28 observations from these works into our analysis, and we describe these sources in further detail in Section 3.4.

Our final SMA follow-up campaign sample consisted of 75 total targets; 23 in the UDS field, nine in the SSA22 field, 16 in the COSMOS field, 18 in the LHN field and nine in the EGS field. These sources had the highest $850 \mu \mathrm{m}$ flux densities down to approximately $10 \mathrm{mJy}$, except in the UDS field where we probed sources with flux densities down to about $8 \mathrm{mJy}$. In Fig. 1 we show the SCUBA-2 deboosted flux density distribution from our parent CLS sample, with the distribution of our targets and the distribution of our full catalogue (including archival sources) overlaid. This shows the completeness of our selection, which we quantify later in Section 3.4 .

We note that we followed up two sources in the EGS field (EGS07 and EGS09) as well as two sources in the COSMOS field (COSMOS01 and COSMOS02) that ended up excluded from the final CLS catalogue. These four sources lie near the edge of the EGS and COSMOS maps, where the root mean square (rms) is higher, and were thus ex- 
cluded from the area used to define the final CLS regions. COSMOS02 is confirmed to be the brightest SCUBA-2 CLS source in a follow-up program to achieve deeper imaging in the COSMOS field (Simpson et al. in prep.), so it's clear that there is some interest in these sources. While these four sources do not appear in our study, we nonetheless report them here for completeness.

\subsection{SMA observations}

We targeted 79 bright SCUBA-2 sources from the CLS fields using the SMA, carried out over a period of two years between November 2014 and November 2016. The sources are widely spaced on the sky and there was never an opportunity to have more than one source within the primary beam of the SMA at $860 \mu \mathrm{m}$. We set up the SMA in the compact configuration tuned to $345 \mathrm{GHz}$ with a bandwidth of at least $4 \mathrm{GHz}$ and had available between six and eight $6-\mathrm{m}$ dishes for a given track. The upgrade of the SMA with the SWARM correlator during this period led to a steady increase in bandwidth during the course of our observing program, culminating in the final track using the full $32 \mathrm{GHz}$ of SWARM. This upgrade considerably improved the continuum sensitivity and made calibrations with fainter quasars easier as the program went on. We adopted track sharing, typically three sources per track, to provide the best possible $u v$-plane coverage of each source (given the limited number of antennas available with the SMA), with some sources repeated on multiple tracks to achieve our desired sensitivity. The synthesized beam achieved in this set up was on average 2.4 arcsec full-width at half-maximum (FWHM) with our natural weighting of the visibilities, but the beam shape in some cases ranged in elongation on the major axis to 3.5 arcsec.

We performed the calibration and data inspection using the IDL-based Caltech package MIR modified for the SMA. We generated continuum data by averaging the spectral channels after doing the passband phase calibration. We used both gain calibrators to derive gain curves. For consistency checks, we compared these results with those obtained by adopting just one calibrator. We did not find any systematic differences. We computed the fluxes using calibrators observed on the same day and under similar conditions (time, hour angle, and elevation). Flux densities were calibrated using typically Uranus, Neptune, Callisto or Titan, depending on availability and proximity to the given target. The flux calibration error is typically within $\sim 10 \%$. Observations ranged in conditions but typically had PVW significantly less than $2 \mathrm{~mm}\left(\tau_{225 \mathrm{GHz}}<0.12\right)$. Our general goal was to detect 100 per cent of a target's SCUBA-2 flux density at $4 \sigma$, however the resulting sensitivities on a given source were mainly determined by scheduling, weather, and available antennas on a given night.

\subsection{Source detections}

We exported the calibrated interferometric visibility data to the package MIRIAD for subsequent imaging and analysis. We weighted the visibility data inversely proportional to the system temperature and Fourier transformed them to form images. We used natural weighting to maximize the signal-to-noise $(\mathrm{S} / \mathrm{N})$. We CLEANed the images around detected sources to approximately 1.5 times the noise level to remove the effects of sidelobes (the results were not sensitive to choosing a slightly deeper CLEANing level, such as 1.0 times the noise). We typically achieved an rms between 1 and $2 \mathrm{mJy}$, but occasionally we were substantially deeper than this with very good weather and SWARM working well. We corrected the images for the SMA primary beam response.

We set a detection threshold of $>4 \sigma$ peaks in our maps. We measured source positions and flux densities by fitting the peaks in the dirty images, and also fitting the images with point-source models using the MIRIAD imfit routine. The results of both approaches were very consistent, and we adopted the former for further analysis. All of the SMA flux densities and flux density errors that we quote henceforth are primary-beam corrected.

In the UDS field we detected 21 out of the 23 SCUBA-2 sources we followed-up; none of these 21 sources were seen to break up into two components, and two sources remained undetected. Within the COSMOS field, our SMA observations detected a total of 13 galaxies from the 16 SCUBA-2 sources: one source broke up into two galaxies; and in four sources we found no peaks greater than $4 \sigma$. Of the nine SCUBA-2 sources targeted in the SSA22 field, four were not detected above the $4 \sigma$ level in the SMA maps, and in the remaining five we found single galaxies. In the LHN field we found 18 galaxies from our targeted sample of 18 SCUBA-2 sources. Of these 18 detections two are SCUBA-2 sources that break up into two galaxies, and in two cases we did not find any galaxies. In the EGS field we have detected single galaxies for all nine SCUBA-2 sources. We also report detections of all four of the SCUBA-2 sources we followed up outside of the boundary of the CLS regions, and note that none resolved into multiples. In addition, COSMOS02, the brightest SCUBA-2 source, was also found to be the brightest source in our SMA observations.

Overall we detected 66 submm galaxies in 75 SMA pointings above a $4 \sigma$ depth of about $6 \mathrm{mJy}$. These detections are summarized in Tables A1-A5, where we provide the positions of both the SCUBA-2 sources and our SMA detections, the measured and deboosted SCUBA-2 flux densities of each target as $S_{\mathrm{S} 2}^{\text {obs }}$ and $S_{\mathrm{S} 2}$, respectively, and our measured flux densities as $S_{\text {SMA }}^{\text {obs }}$. For undetected sources, we report the $4 \sigma$ flux density limit achieved by our observations instead. In each field, we sort sources in descending order of their deboosted SCUBA-2 flux density.

\subsection{Source IDs}

In Fig. 2 we show SMA contours of eight representative samples in the UDS field overlaid over existing Spitzer-IRAC $3.6 \mu \mathrm{m}$, VLA $1.4 \mathrm{GHz}$ and parent SCUBA-2 $850 \mu \mathrm{m}$ images. We can see that there are IR/radio sources coincidental with nearly all of our SMA positions to within 1 arcsec, yielding robustly identified counterpart galaxies. The multiwavelength properties of these galaxies will be investigated in future work.

It is worth noting that in the COSMOS field, out of the 18 SCUBA-2 sources found by Michałowski et al. (2017) to have multiwavelength counterparts and included in our sample, all were confirmed by our SMA imaging. In the UDS 
field, out of 35 SCUBA-2 sources overlapping between our two studies, 31 were confirmed (89 per cent), consistent with the reliability of $\simeq 92$ per cent measured by Michałowski et al. (2017) based on the ALMA data of Simpson et al. (2015). Similarly, Chen et al. (2015) was able to identify multiwavelength counterparts for $\simeq 79$ per cent of the SCUBA-2 sources detected in the Extended Chandra Deep Field South, consistent with our observations.

\section{ANALYSIS}

\subsection{Flux boosting}

The effects of selection biases, particularly 'flux boosting', on our results are complicated. This is because we picked bright outliers in large SCUBA-2 maps and followed them up with the SMA at higher resolution. Because of this complexity, we put considerable effort into simulating our observing and analysis procedure. The effect of flux boosting results from the statistical nature of measuring flux densities in a noisy map where there are many more faint sources than bright ones. This effect will tend to scatter sources to higher flux densities rather than lower ones, hence the term 'boosting'. One approach to correct for flux boosting follows from Bayes' theorem:

$$
P\left(S_{\text {true }} \mid S_{\text {obs }}, \sigma_{\text {obs }}\right) \propto P\left(S_{\text {true }}\right) P\left(S_{\text {obs }} \mid S_{\text {true }}, \sigma_{\text {obs }}\right),
$$

where $S_{\text {true }}$ is the intrinsic source flux density, $S_{\text {obs }}$ is the measured source flux density and $\sigma_{\mathrm{obs}}$ is the measured source uncertainty. If the source uncertainties are Gaussian then

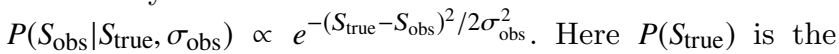
prior, which quantifies all previous knowledge that we might have about the distribution of flux densities in our sample.

To construct the prior we performed a set of simulations that reconstruct, as best as possible, our observing strategy. For each of the five fields in our study we first produced a mock SCUBA-2 map with $2 \operatorname{arcsec} \times 2$ arcsec pixels by injecting sources into an area of blank sky matching the area surveyed in the SCUBA-2 CLS. The flux densities were drawn from a Schechter-type function of the form

$\frac{d N}{d S}=\left(\frac{N_{0}}{S_{0}}\right)\left(\frac{S}{S_{0}}\right)^{-\gamma} e^{-S / S_{0}}$.

We adopted parameters obtained by Casey et al. (2013) from a fit to the number counts in a roughly $0.1 \mathrm{deg}^{2}$ portion of the COSMOS field, namely $N_{0}=3300 \mathrm{deg}^{-2}, S_{0}=3.7 \mathrm{mJy}$ and $\gamma=1.4$. While Geach et al. (2017) also fit this model to their number counts, we found that the above values were more consistent with our data as they predicted more bright sources. Positions were randomly selected to simulate Poisson statistics, with no clustering. The maps were convolved with a nominal SCUBA-2 beam with a FWHM of 14.8 arcsec and a negative bowl (from Geach et al. 2017). Gaussian noise was added followed by a second smoothing with the SCUBA-2 beam, which is the matched filter that optimizes point-source detection (see Chapin et al. 2011, Appendix A). The amplitude of the Gaussian noise is such that, after application of the matched filter, the resulting rms is equivalent to the rms achieved in each fields' actual map. We note that in practice the SCUBA-2 noise is not Gaussian and is correlated; however, the SCUBA-2 CLS areas were covered sufficiently uniformly that our method does not introduce significant errors.

To simulate the SMA follow-ups, we found all peaks in the map brighter than a certain cutoff, which was determined to be the faintest non-deboosted SCUBA-2 source targeted by our actual SMA observations in a given field. The mock SMA follow-ups were performed by creating 9 arcsec $\times 9$ arcsec thumbnail images centred on a bright SCUBA2 source's peak pixel; we chose 9 arcsec as a characteristic thumbnail size, since beyond this radius we no longer expect to be seeing the source/sources that contribute to the SCUBA-2 flux density we are following up (see Section 3.2 and Fig. 4). The thumbnail images have 0.1 arcsec pixel sizes and were smoothed by a 2.4 -arcsec FWHM beam, which accurately reconstructed our actual SMA observations because most of the galaxies in our data are unresolved. Following Coppin et al. (2005, 2006), the distribution of pixel flux densities from all of the mock SMA observations is a good estimator for the prior, since it takes into account both resolution and selection effects present in our observations. For each of the five fields where we have data, we repeated our simulation a sufficient number of times to produce $100 \mathrm{deg}^{2}$, corresponding to over 70,000 sources brighter than $10 \mathrm{mJy}$. The parameters used in each of the five fields' simulations are summarized in Table 1.

Following Eq. 1, we constructed a posterior probability distribution for the intrinsic flux density of each source using priors from their respective fields. In Tables A1-A5 under the column $S_{\mathrm{SMA}}$ we report the deboosted flux density as the peak in the posterior probability distribution, and we give error bars representing 68 per cent confidence intervals. In Fig. 3 we show an example of this deboosting technique for a typical source, COSMOS14, which, according to our simulations, is expected to be 4 per cent fainter than indicated by our maps. Note that the error bars do not necessarily increase, but the signal always decreases so that the $\mathrm{S} / \mathrm{N}$ always decreases. We also note that COSMOS22, which had a $\mathrm{S} / \mathrm{N}$ value just at the threshold of 4.0 , had a probability density function that also peaked at zero flux density, so we report a 68 per cent upper limit for this source as well.

Cases where a single bright SCUBA-2 source is resolved into two or more faint galaxies are more difficult to deboost. In our simulations we do not include any galaxygalaxy interactions, clustering or lensing, and we only followup the SCUBA-2 sources brighter than a certain threshold, so we cannot use our approach to obtain deboosting fractions for those faint galaxies which contribute to single, bright SCUBA-2 peaks. However, should a bright SCUBA2 source resolve into one bright SMG above our follow up threshold and one or more faint SMGs below our follow up threshold, our boosting correction would be applicable only to the bright SMG. We therefore define all faint galaxies to be those with flux densities $1 \mathrm{mJy}$ less than the cutoff used to determine which SCUBA-2 sources were to be followed up by the SMA in our simulations in a given field. Galaxies LHN13a and LHN13b resolved completely from a SCUBA-2 peak and are considered faint, while COSMOS11b resolved from a SCUBA-2 peak along with a bright companion. We did not correct the measured flux densities for these SMGs, and we simply use the measured values throughout the paper; in the $S_{\mathrm{SMA}}$ column of Tables A1-A5, we report a value 
UDS16

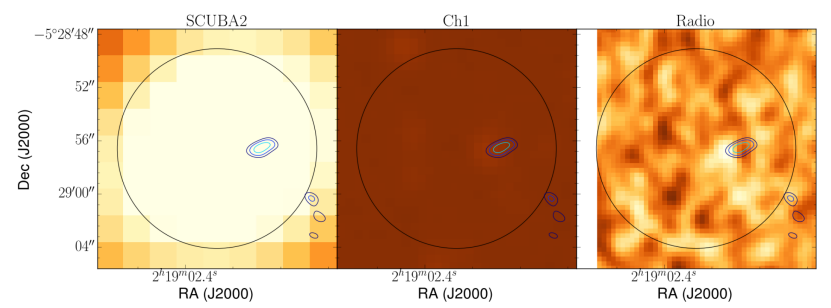

UDS22

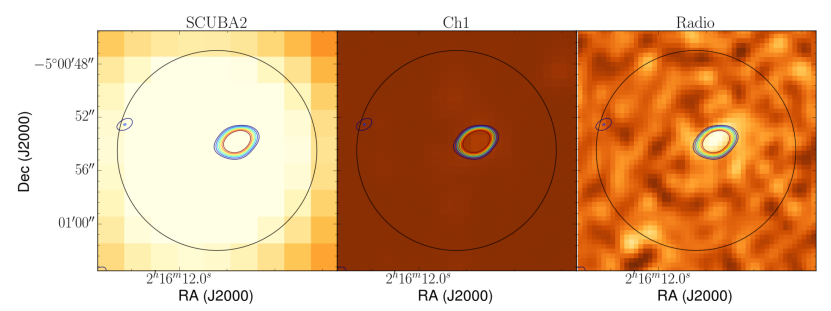

UDS29

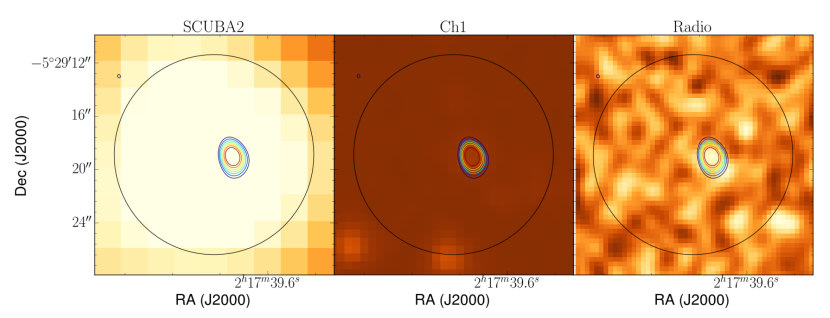

UDS37

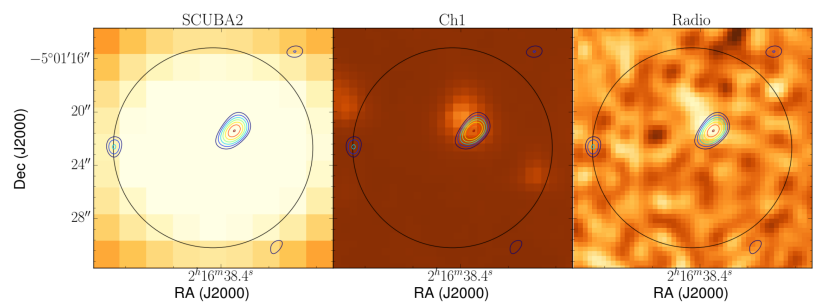

UDS20

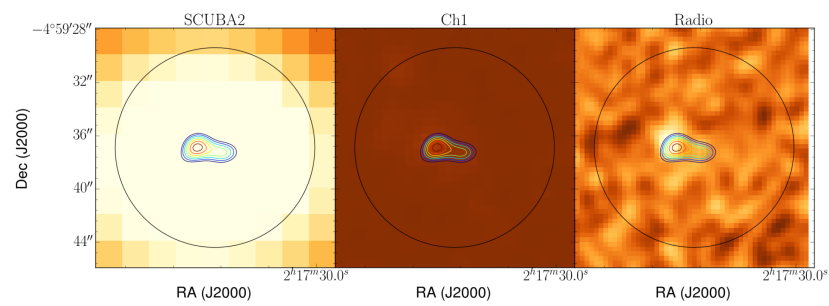

UDS28

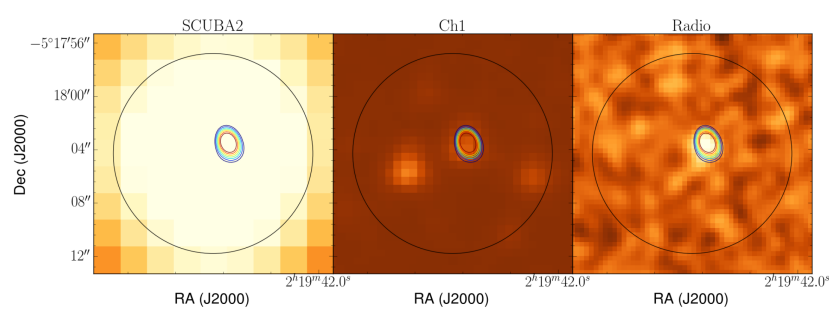

UDS34

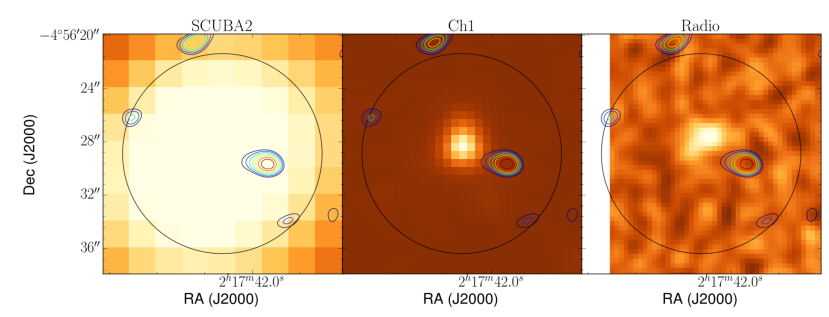

UDS39

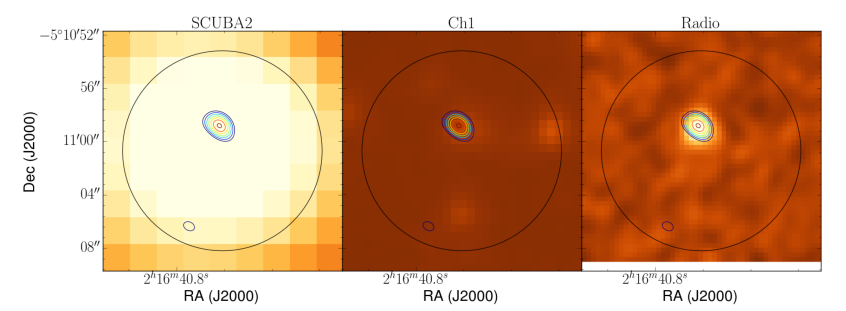

Figure 2. Multiwavelength cut-outs of a subset of eight representative UDS sources in our sample with existing Spitzer-IRAC 3.6 $\mu$ m and VLA $1.4 \mathrm{GHz}$ imaging. We show SMA flux contours ranging from $3 \sigma$ to $6 \sigma$ in steps of $0.5 \sigma$ overlaid over the IR and radio data, plus the parent SCUBA-2 $850 \mu \mathrm{m}$ data. Also shown is a 14.8 arcsec circle representing the SCUBA-2 beam.

Table 1. Parameters describing our simulations, which we use to calculate the expected level of flux boosting in our measurements.

\begin{tabular}{lccc}
\hline Field & $\begin{array}{c}\text { S2 area } \\
{\left[\mathrm{deg}^{2}\right]}\end{array}$ & $\begin{array}{c}\text { S2 noise } \\
{[\mathrm{mJy}]}\end{array}$ & $\begin{array}{c}\text { S2 cutoff } \\
{[\mathrm{mJy}]}\end{array}$ \\
\hline UDS & 0.96 & 0.9 & 7.8 \\
SSA22 & 0.28 & 1.2 & 6.7 \\
COSMOS & 2.22 & 1.6 & 7.2 \\
LHN & 0.28 & 1.1 & 8.1 \\
EGS & 0.32 & 1.2 & 9.8 \\
\hline
\end{tabular}

of N/A for these cases. We note that neglecting to deboost these faint sources will have no effect on the bright end of the number counts.

\section{$3.2 \quad$ Astrometry}

The accuracy with which SCUBA-2 sources can be localized is well understood to be a function of observed S/N (assuming no multiplicity), and is approximated as (equation B22 in Ivison et al. 2007)

$\Delta \alpha=\Delta \delta=0.6$ FWHM $\left[(\mathrm{S} / \mathrm{N})_{\text {obs }}^{2}-(2 \beta+4)\right]^{-1 / 2}$,

where FWHM is the beamsize of SCUBA- 2 and $\beta$ is the local slope of the cumulative number count used as a prior to correct the observed flux densities for boosting. To examine the positional accuracy of our sample we computed the radial distance between our interferometrically-detected sources and those of the parent SCUBA-2 catalogue as a function of the detected S/N from Geach et al. (2017). For cases 


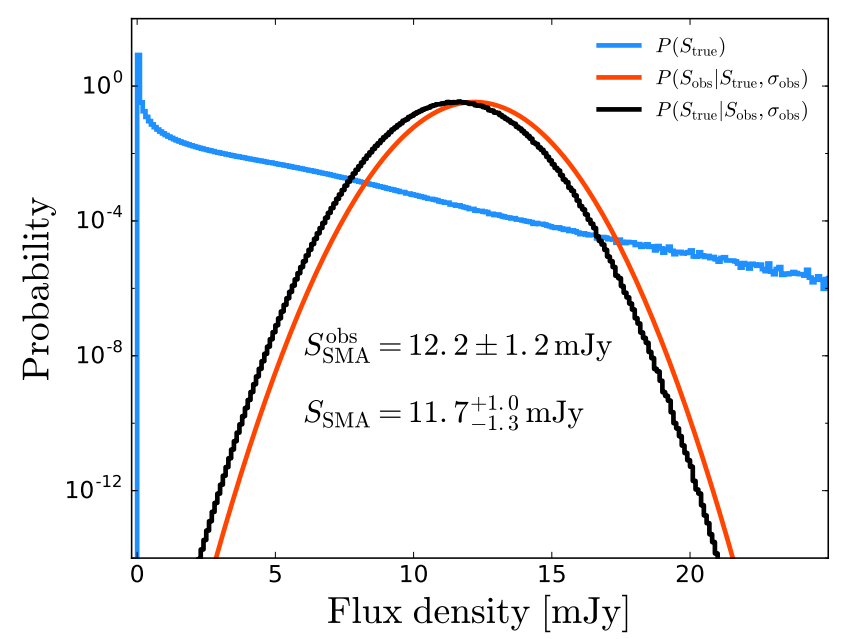

Figure 3. Probability distributions for the flux density of COSMOS14, a typical source in our data set. The blue curve is the prior, which is calculated by binning pixels resulting from simulating SCUBA-2 CLS fields and making small SMA thumbnail images centred on the brightest sources. The red curve shows the flux density of COSMOS14 measured from our data, where the uncertainty is assumed to be Gaussian. The black curve is the posterior probability distribution, which peaks at a slightly lower, deboosted flux density value due to the presence of many more faint galaxies in the simulated sky. The deboosted flux density uncertainties given represent a 68 per cent confidence interval about the peak.

where multiple SMA/ALMA sources are detected, we simulated a simple (noiseless) SCUBA-2 image by convolving point sources at the SMA positions with a nominal SCUBA2 beam with a FWHM of 14.8 arcsec (the best-fitting model from Geach et al. 2017, resulting from stacking 322 point sources of $>5 \sigma$ in the UDS map) and calculated the location of the peak intensity, which is then compared to the reported SCUBA-2 source position. We took into account offsets between the SMA and SCUBA-2 reference frames on a field-by-field basis by subtracting the mean difference in right ascension and declination from each calculated offset.

In Fig. 4 we plot the radial separation of our SMA positions relative to the SCUBA-2 positions (except for the 13 sources where we did not detect a galaxy) as a function of detected S/N. Also shown are theoretical 68 per cent and 95 per cent contours, derived using Equation 3 with $\beta=2.4$. To obtain the radial probability density we integrate $r e^{-r^{2} / 2 \sigma^{2}}$, so 68 per cent and 95 per cent contours are are actually at $1.51 \sigma$ and $2.50 \sigma$, respectively. Six sources lie above the 95 per cent contour, corresponding to about 10 per cent of the sources in our sample, which is only marginally more than expected. There also appears to be one outlier with a 9 arcsec offset. This outlier is LHN09. In Fig. 5 we show SMA contours for this source overlaid over Spitzer-IRAC $3.6 \mu \mathrm{m}$, VLA $1.4 \mathrm{GHz}$ and the parent SCUBA-2 $850 \mu \mathrm{m}$ images. We can see that LHN09 is a rather complicated system, with at least four IR galaxies and two radio galaxies all within the SCUBA-2 beam. It is therefore possible that our SMA data has either resolved out some of the flux density seen by SCUBA-2 or that most of these multiwavelength coun-

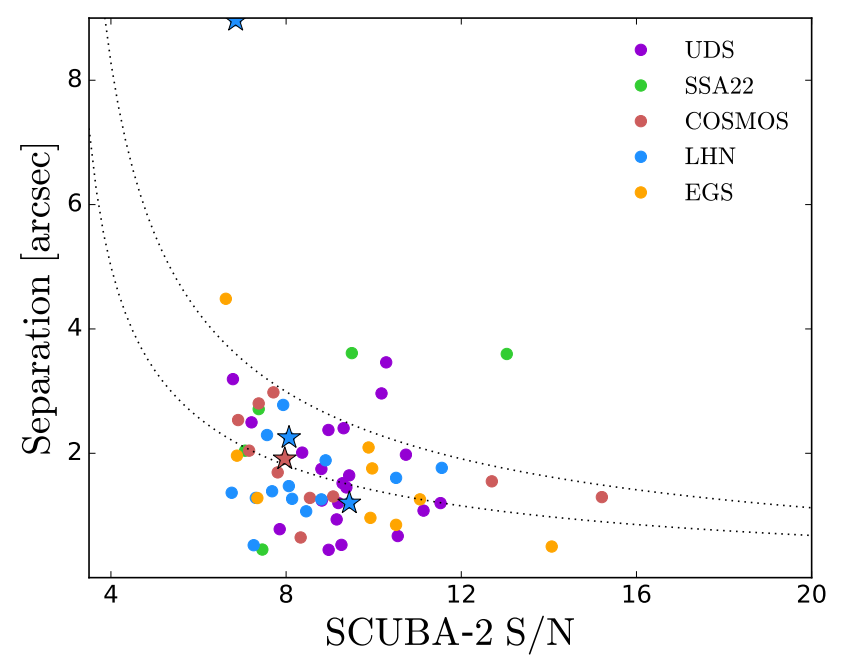

Figure 4. Radial offset of SMA-detected sources from their SCUBA-2 counterparts. Where multiple counterparts are detected we smooth the sources with the nominal SCUBA-2 beam and locate the peak flux density and compare this to the given SCUBA-2 position. These sources are highlighted in the figure by stars. Also shown are the expected 68 per cent and 95 per cent positional uncertainties as a function of detected $\mathrm{S} / \mathrm{N}$ for SCUBA-2.

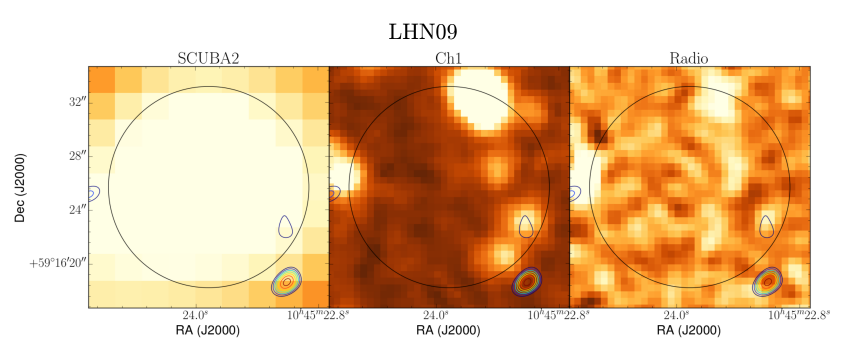

Figure 5. SMA flux density contours of LHN09, from $3 \sigma$ to $6 \sigma$ in steps of $0.5 \sigma$, overlaid over Spitzer-IRAC $3.6 \mu \mathrm{m}$, VLA $1.4 \mathrm{GHz}$ and SCUBA-2 $850 \mu \mathrm{m}$ data. We see a large offset between the peak SCUBA-2 $850 \mu \mathrm{m}$ flux density and the SMA detection. This could be due to the fact that this is a rather complicated field, with at least four IR galaxies and two radio galaxies all within the SCUBA-2 beam.

terparts are fainter than out flux density limit, leading to the large offset that we are seeing.

\subsection{Flux density reliability}

Next we compare the interferometric flux density observations to those from SCUBA-2 to check the reliability of the flux densities in our data set. We use the boostingcorrected flux densities reported by Geach et al. (2017) and our boosting-corrected flux densities. When comparing the cases where a singe-dish source is resolved into multiple components, we take into account the SCUBA-2 14.8 arcsec beamsize. To do this, for each component, we multiply the flux density by a 14.8-arcsec FWHM Gaussian, representing the SCUBA-2 beam, evaluated at the angular separation between the component and the location of the peak 


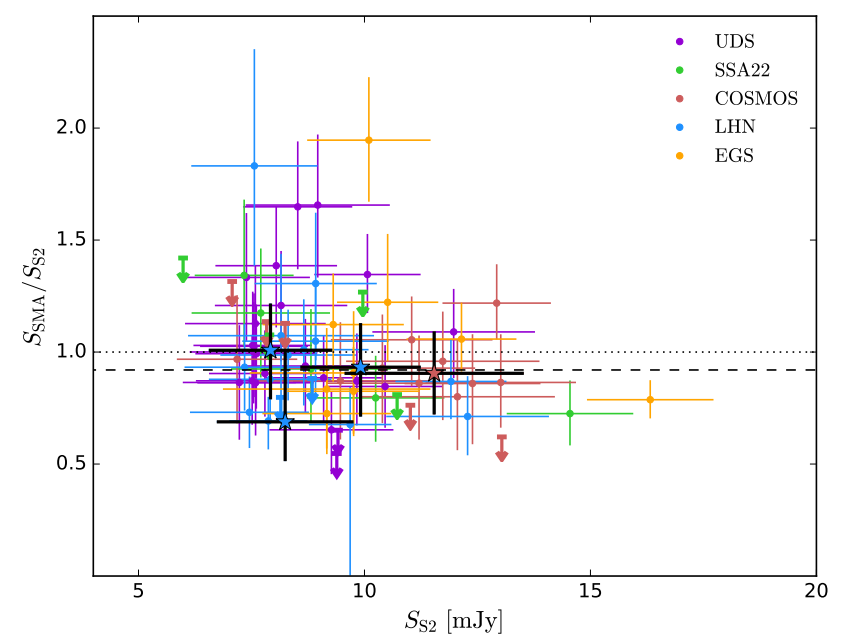

Figure 6. Comparison of the SCUBA-2 deboosted flux density from Geach et al. (2017) to the ratio of our SMA deboosted flux densities to each corresponding SCUBA-2 flux density. Where a single CLS source is resolved into multiple components, we have summed each components' flux density weighted by the SCUBA2 beam response. These sources are shown as stars. Targets were we were only able to determine upper limits on the SMA flux density are shown as downward pointing arrows. The dotted line indicates a flux ratio of 1 , expected if we recovered 100 per cent of the SCUBA-2 flux density, and the dashed line shows our median ratio of $0.93_{-0.03}^{+0.05}$, which could be less than 1 due to the presence of faint galaxies below the sensitivity of our observations.

flux density seen in the SCUBA-2 map. Summing up these weighted flux densities is a better approximation to what was observed by SCUBA-2. The results are shown in Fig. 6, where we have plotted $S_{\mathrm{S} 2}$ versus $S_{\mathrm{SMA}} / S_{\mathrm{S} 2}$. In this figure the multiples are shown as stars and the 13 sources where we were only able to obtain upper flux density limits in our SMA observations are shown as downward pointing arrows.

The median value of the ratio $S_{\mathrm{SMA}} / S_{\mathrm{S} 2}$, not including the 13 blank maps, is $0.93_{-0.03}^{+0.05}$, where the uncertainty was calculated as the 68 per cent confidence interval from bootstrap resampling. This result shows that there is evidence for some missing flux from fainter sources in our maps with detections, but it is likely that there are also faint sources below the noise levels in our maps lacking detections. When we add the ratios of $S_{\mathrm{SMA}} / S_{\mathrm{S} 2}$ from these blank maps, calculated using the upper limits derived for $S_{\mathrm{SMA}}$, the median value of $S_{\mathrm{SMA}} / S_{\mathrm{S} 2}$ is $0.93_{-0.03}^{+0.03}$, which has the same value but smaller bootstrap uncertainty, which is still consistent with the interpretation that there are faint galaxies below our noise thresholds.

\subsection{Completeness}

Here we discuss in detail the completeness of our observations with respect to the parent SCUBA-2 CLS survey. Since our targets were selected based on early CLS maps with higher noise, it is important to understand our sample in terms of the final, published maps. In addition, we have to decide how many sources from other experiments (i.e. Younger et al. 2007, 2009; Simpson et al. 2015) we wish to include in our sample, since they will affect our completeness.

The latter question is important because we have not specifically targeted any sources below the given flux density limit (which depends on the field); however, images from Younger et al. (2007, 2009) and Simpson et al. (2015) extend much deeper, and in several cases a faint source that would have been omitted from our study turned out to be bright enough to affect the bright end of the number counts when observed by the SMA or ALMA. Including sources like this could potentially bias our results, since our survey would then not really be 'blind', rendering the analysis much more difficult to interpret.

Our approach to this problem involves two steps. First, we incorporate into our catalogue all sources from Younger et al. (2007, 2009) and Simpson et al. (2015) that have SCUBA-2 deboosted flux densities greater than the faintest source we targeted in our observations in a given field (see Table 1, under the 'S2 thresh' column). These sources are included in Tables A1-A5, and we have used the numbering conventions given in their respective papers. There are seven sources from Younger et al. (2007), four sources from Younger et al. (2009) and 16 sources from Simpson et al. (2015), for a total of 27 archival sources. We note that the flux densities from Younger et al. $(2007,2009)$ have not been corrected for flux boosting, so we use their direct measurements and give the deboosted flux densities under the $S_{\mathrm{SMA}}$ column as N/A; the flux densities from Simpson et al. (2015) have been corrected for flux boosting, which are given under the $S_{\text {ALMA }}$ column, and we use these values for further analysis. We also include in our work the SMA observation of Orochi from Ikarashi et al. (2011), the gravitational lens in the UDS field. This brings the total number of interferometric samples in our analysis to 103 .

Next, we calculate a completeness level for that field by dividing the total number of SCUBA-2 sources targeted in our sample by the total number of SCUBA-2 sources in the parent sample in a given flux density bin. We looked at bins above $8 \mathrm{mJy}$ with widths of $\Delta S=1 \mathrm{mJy}$. In this way we are effectively treating the external sources as if we had targeted them ourselves, introducing as little bias as possible, while still using all of the data. We can then use the calculated completeness values in each bin to correct for the missing sources introduced in the final, deeper CLS SCUBA-2 maps.

In the UDS field, we have targeted sources down to SCUBA-2-deboosted flux densities of $7.2 \mathrm{mJy}$. After introducing the sources from Simpson et al. (2015) with deboosted SCUBA-2 flux densities greater than $7.2 \mathrm{mJy}$, we find that our catalogue reaches a completeness of 96 per cent for $S_{850}>8 \mathrm{mJy}$, where the unobserved 4 per cent of sources are cases where a SCUBA-2 flux density was scattered to a higher value with the additional exposure time. At fainter flux densities our completeness falls below 80 per cent, which we deem to be too low to be used reliable, and in the brighter regime of $S_{850}>9$ mJy we have 100 per cent completeness. A similar analysis performed for the ALMA sources observed by Simpson et al. (2015) resulted in completeness levels of 50 per cent for $S_{850}>8 \mathrm{mJy}, 56$ per cent for $S_{850}>9 \mathrm{mJy}$, and 73 per cent for $S_{850}>10 \mathrm{mJy}$, which shows that our observations offer a significant improvement in this field owing to the fact that our targets were selected from later versions of the CLS maps. 
Table 2. Completeness levels calculated for each field in our study, as well as for the total data set.

\begin{tabular}{lcc}
\hline Field & $\begin{array}{c}\text { Completeness } \\
S_{850}>8 \text { mJy }\end{array}$ & $\begin{array}{c}\text { Completeness } \\
S_{850}>10 \mathrm{mJy}\end{array}$ \\
\hline UDS & $96 \%$ & $100 \%$ \\
SSA22 & $71 \%$ & $100 \%$ \\
COSMOS & $54 \%$ & $89 \%$ \\
LHN & $100 \%$ & $100 \%$ \\
EGS & $\mathrm{N} / \mathrm{A}$ & $100 \%$ \\
\hline Total & $77 \%$ & $95 \%$ \\
\hline
\end{tabular}

In the SSA22 field we have followed up 100 per cent of the sources with a deboosted SCUBA-2 850- $\mu$ m flux density greater than $10 \mathrm{mJy}$. In this field there are no sources with SCUBA-2 850- $\mu \mathrm{m}$ deboosted flux densities between 9 and $10 \mathrm{mJy}$, and below $9 \mathrm{mJy}$ our data do not cover enough sources to allow us to reliably estimate the number counts. Despite the fact that we have targeted five additional sources less than the $S_{850}=10 \mathrm{mJy}$ level, two sources scattered up to about $8 \mathrm{mJy}$ in the deeper SSA22 $850 \mu \mathrm{m}$ CLS map after our targets were selected, and so our completeness for $S_{850}>8 \mathrm{mJy}$ is only 71 per cent.

In the COSMOS field, only about 50 per cent of the total area was mapped to a nominal depth of $1.6 \mathrm{mJy}$ in the published $850 \mu \mathrm{m}$ CLS maps used in our study, and the remaining half is currently being completed (S2COSMOS: Simpson et al. in prep.); our completeness calculation for this field is based on the current data available in Geach et al. (2017). We find that, with the addition of the observations from Younger et al. $(2007,2009)$ down to $7.1 \mathrm{mJy}$, our faintest target, we have completeness of 89 per cent for $S_{850}>10 \mathrm{mJy}$, and 100 per cent completeness for $S_{850}>11 \mathrm{mJy}$. Below $10 \mathrm{mJy}$ our sample becomes very sparse. There are two sources with deboosted SCUBA-2 $850 \mu \mathrm{m}$ flux densities of 10.0 and $10.1 \mathrm{mJy}$ that have not been observed with the SMA in our campaign, nor in the work of Younger et al. (2007, 2009), due to their low S/N in earlier SCUBA-2 and LABOCA maps.

We have fully probed the LHN field down to $S_{850}=7.5 \mathrm{mJy}$, achieving 100 per cent completeness. Below this we targeted one source whose corresponding deboosted SCUBA-2 $850 \mu \mathrm{m}$ flux density is $7.3 \mathrm{mJy}$, but we do not try to probe number counts this low.

Lastly, our sample does not include any EGS members with $S_{850}<9 \mathrm{mJy}$, while for $S_{850} \geq 9 \mathrm{mJy}$ we have resolved all of the available CLS sources, and thus every detection is statistically significant for estimating the counts in this field.

We now consider the completeness of our total data set. We have observed nearly all sources down to $850 \mu \mathrm{m}$ flux densities of $10 \mathrm{mJy}$ in these five cosmological fields, reaching a completeness level of 95 per cent for $S_{850}>10 \mathrm{mJy}$. As described above, there are two SCUBA-2 sources with deboosted flux densities at $850 \mu \mathrm{m}$ of 10.0 and $10.1 \mathrm{mJy}$ that have no interferometric data, both in the COSMOS field. When considering our full data set, these two sources comprise 5 per cent of the total number of sources with $S_{850} \geq 10 \mathrm{mJy}$. In Table 2 we summarize our completeness calculations for each field, for $S_{850}>8 \mathrm{mJy}$ and $S_{850}>10 \mathrm{mJy}$.

\section{RESULTS AND DISCUSSION}

\subsection{Number counts}

We now estimate the cumulative number counts of our sample of interferometrically-detected SMGs. Our calculations are restricted to counts within the completeness regimes discussed above. The areas for each field are given in Geach et al. (2017) and are $0.96 \mathrm{deg}^{2}$ for the UDS field, $0.28 \mathrm{deg}^{2}$ for the SSA22 field, $2.22 \mathrm{deg}^{2}$ for the COSMOS field, $0.28 \mathrm{deg}^{2}$ for the LHN field and $0.32 \mathrm{deg}^{2}$ for the EGS field, totalling $4.06 \mathrm{deg}^{2}$ for our complete survey. We calculate the cumulative number count in bins of $\Delta S=1 \mathrm{mJy}$ by simply counting the total number of sources $>S$ and dividing by the total area. To correct for incompleteness at the faintest flux density bin $(10 \mathrm{mJy})$ used in our calculations, we multiplied the total area by the fraction of sources targeted in our survey relative to the sources in the parent CLS catalogue (i.e. the completeness).

For the 13 observations where only upper limits were obtained for the SMA counterparts we use the upper limit flux density as the deboosted SMA flux density; all $4 \sigma$ upper limits we have measured constrain the flux densities of these sources to $S_{860}<10 \mathrm{mJy}$, below the regime where we are calculating the counts, so we are not introducing any bias in the flux density region studied in this work by doing this. The source SSA22-04 is however an exception, where we have constrained the flux density to be less than 12.6 mJy but the corresponding SCUBA-2 flux density is $10.0 \mathrm{mJy}$. Since our SMA observations of this source have not been able to provide any further information, we have removed this source from our calculation and corrected for the incompleteness this introduces using the procedure described above. Lastly, for plotting purposes, we remove all repeated points, that is, points where there is no change in the cumulative number count in two adjacent bins because there are no sources between $S$ and $S+\Delta S$.

The results for the cumulative number count are shown in Fig. 7. The error bars are calculated as 68 per cent confidence intervals from Poisson statistics (see Gehrels 1986). In addition, we show the CLS cumulative count results from Geach et al. (2017) for comparison. We have also shaded the boundary marking the 100 per cent completeness of our sample.

We then compute the differential number counts in each field, following the same procedure as above. The results are shown in Fig. 7, beside our cumulative number counts and together with the CLS differential counts from Geach et al. (2017) and the region marking the boundary of 100 per cent completeness.

In Fig. 8 we show our cumulative and differential number counts for the UDS field alone compared to those derived by Simpson et al. (2015), along with the shaded region indicating our 100 per cent completeness limit. There seems to be a slight lack of sources at $S_{850} \gtrsim 10 \mathrm{mJy}$ seen by Simpson et al. (2015), but this is probably due to incompleteness in their data; there are three SCUBA-2 sources (UDS03, UDS08 and UDS09) that were not targeted in their work as they did not appear to among the brightest 30 UDS sources in the earlier, shallower CLS maps used to design their follow-up ALMA programme. Also shown in Fig. 8 is the cumulative and differential count from the SCUBA-2 data in Geach et al. (2017). By including the three bright 

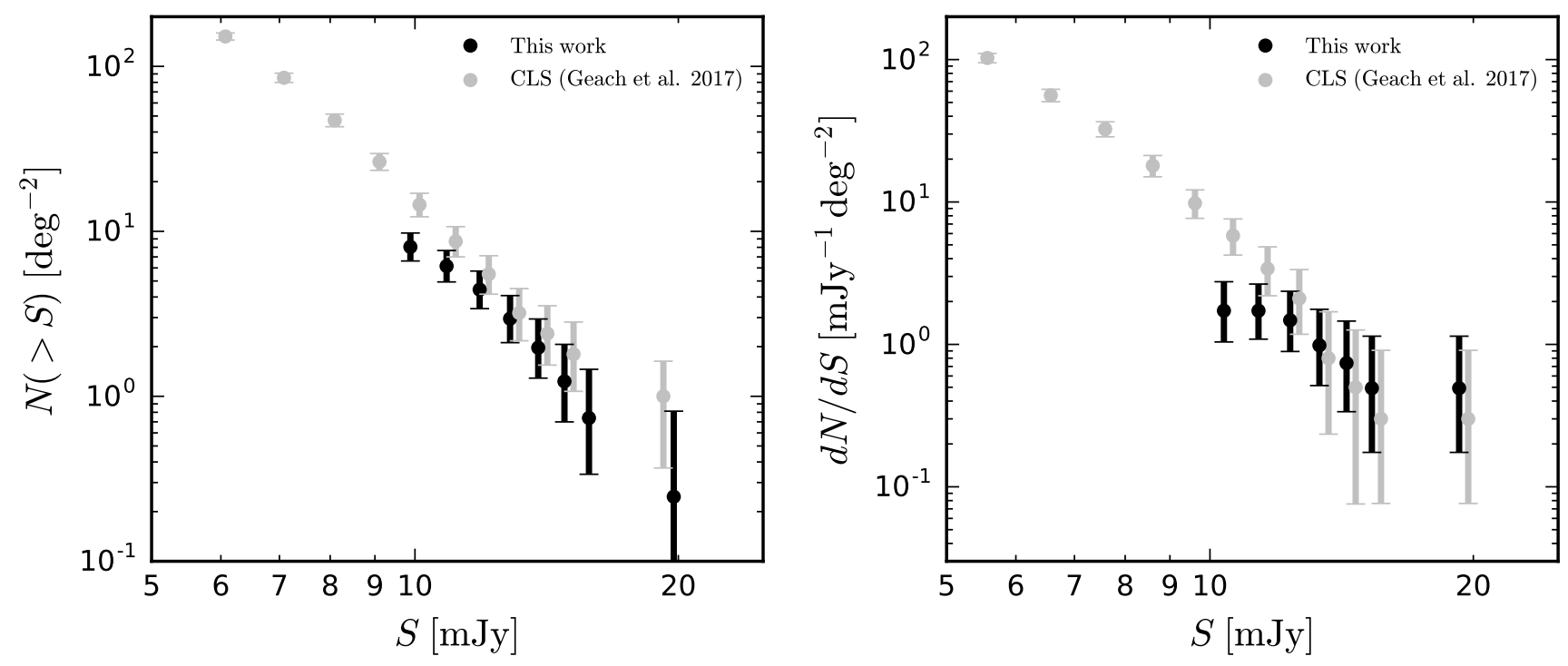

Figure 7. Cumulative and differential number counts derived from our data set. The single dish results from the CLS (Geach et al. 2017) are shown for comparison. Values are slightly offset from each other in each bin for clarity. The shaded region marks where our data is no longer 100 per cent complete. An offset between our results of 20 to 30 per cent is seen in the cumulative count, although the points overlap within the uncertainties.

UDS sources to the number counts we find no strong evidence for diagreement between the single-dish measurements from Geach et al. (2017), the measurements from Simpson et al. (2015) and our work within the uncertainties.

Similar single dish counts were also obtained by the LESS survey (Weiß et al. 2009), which was a 0.35- $\mathrm{deg}^{2}$ $870-\mu \mathrm{m}$ survey of the E-CDF-S carried out with LABOCA, which has a FWHM of 19.2 arcsec. The LESS survey detected a total of 126 submm galaxies to a noise level of approximately $1.2 \mathrm{mJy}$. Following this, a high-resolution follow-up campaign was carried out by Hodge et al. (2013) using ALMA, and the number counts were presented by Karim et al. (2013). They found no sources brighter than $S_{870} \simeq 9$ mJy despite there being 12 LABOCA sources in this regime, implying a cut-off to possible FIR luminosities and star-formation rates.

We compare our results to these earlier works in Fig. 9, where on the top row we have plotted the cumulative and differential number counts from LESS and the CLS (i.e. two single dish submm surveys), and on the bottom row we have plotted the cumulative and differential number counts from Karim et al. (2013), Simpson et al. (2015) and our work (i.e. high angular resolution follow-up studies); the shaded region indicating where our data is no longer 100 per cent complete is shown as well. We see no evidence for a lack of high flux density sources, as hinted at by the results of Karim et al. (2013), and instead see the number count carrying on at a relatively constant slope to around $15 \mathrm{mJy}$. In this plot we have included the number counts from models of evolving star-forming galaxies, specifically the empirical model from Béthermin et al. (2012) and the GALFORM model from Lacey et al. (2016).

Lastly, we fit a power law to our differential count in order to quantitatively compare our results with these other works. We fit only points between 11 and $16 \mathrm{mJy}$, since our flux density coverage for smaller values is not 100 per cent complete, and beyond $16 \mathrm{mJy}$ the differential number count begins to flatten, likely due to gravitational lensing not captured by a simple power law. Our model is of the form

$\frac{d N}{d S}=N_{0} S^{-\gamma}$,

and we find best-fit parameters of $\gamma=4.1 \pm 1.9$ and $N_{0}=$ $(0.4 \pm 1.8) \times 10^{5} \mathrm{mJy}^{-1} \mathrm{deg}^{-1}$. This best-fit curve is plotted alongside our data in Fig. 9. We then compute the $\chi^{2}$ value between our model and the two data points between 11 and 16 mJy from Simpson et al. (2015), finding a value of 0.34 . Taking the number of degrees of freedom to be 1 , this corresponds to a $p$-value of 0.56 . A similar analysis for the five data points (so 4 degrees of freedom) from Geach et al. (2017) between 11 and $16 \mathrm{mJy}$ results in a $\chi^{2}$ value of 3.99 and a $p$-value of 0.41 . These $p$-values, being much greater than the commonly used threshold of 0.05 (e.g., Ivison et al. 2002; Pope et al. 2006; Chapin et al. 2009; Wardlow et al. 2011; Yun et al. 2012), do not suggest that the differential measurements from Simpson et al. (2015) and Geach et al. (2017) differ significantly from our best-fit power law model within the flux density range of 11 to $16 \mathrm{mJy}$, although it is worth noting that we have not incorporated the uncertainty of the best-fit parameters in this analysis.

Even though this simple calculation shows that our results and those from the parent CLS sample are largely consistent, it should be noted that the two data sets are entirely correlated, being observations of the exact same galaxies. Thus any differences at all in the counts, even if they are within the Poisson errors, still carry importance. In particular, the cumulative distribution in Fig. 7 shows that our cumulative number count is systematically lower than the parent SCUBA-2 cumulative number count. We calculate the mean fractional difference between the two cumulative 

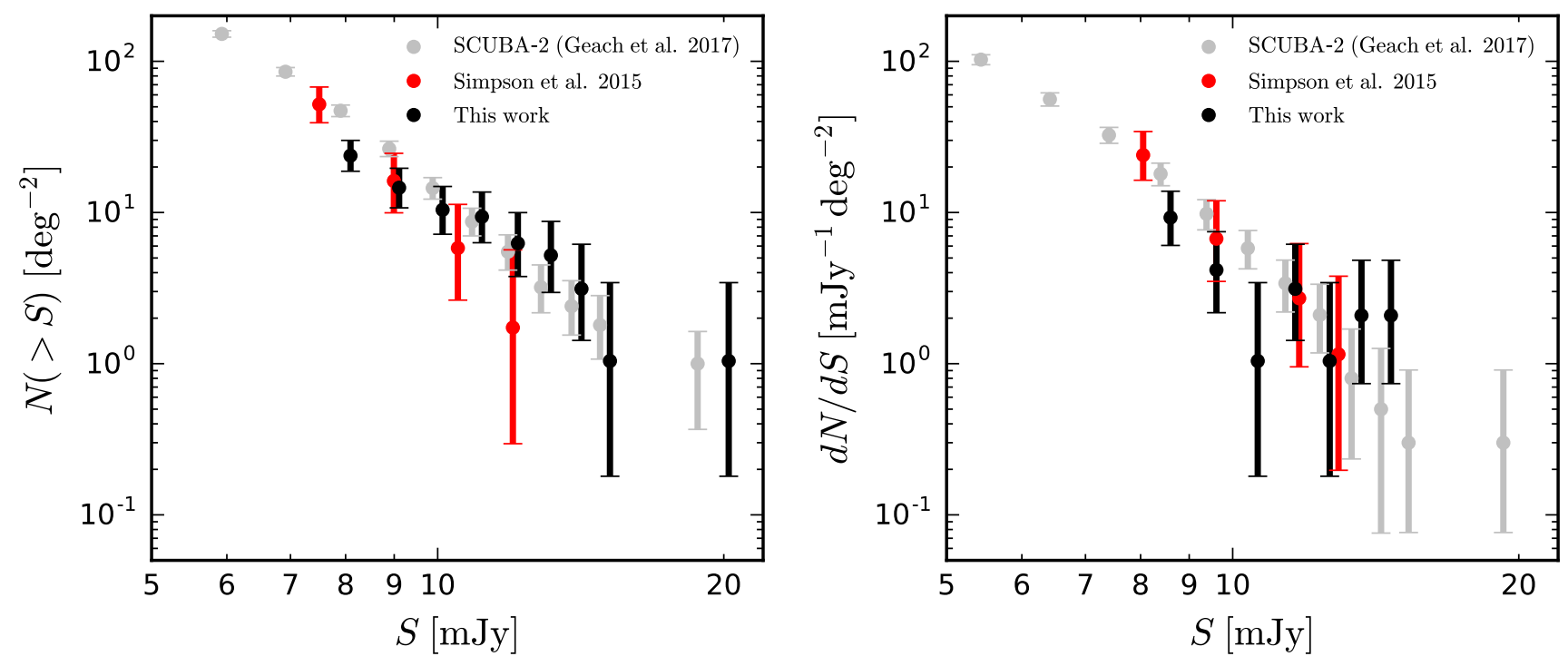

Figure 8. Cumulative and differential number count comparison for the UDS field. The results from Simpson et al. (2015), derived from a smaller sample of the full parent CLS catalogue of the UDS field, are shown in red, alongside our more complete sample in black, where we have used only data from the UDS field as well. The results broadly agree, although we see evidence for less bright sources in the Simpson et al. (2015) sample. Also shown as the shaded region is where our data is not 100 per cent complete; our UDS data is 96 per cent complete for $S>8$ mJy.

number counts between 11 and $15 \mathrm{mJy}$ to be $24 \pm 6$ per cent, where the uncertainty is the standard error of the mean.

\subsection{Multiplicity}

The importance of galaxy interactions and mergers for the intense star-formation rates observed in many submm galaxies is a hotly debated topic. Here we discuss the multiplicity seen in our large sample of bright, $850-\mu$ m-selected galaxies at a resolution of about 2 arcsec, and contrast our observations with previous works.

There is first the question of how to precisely define a multiple; with enough sensitivity, due to the steep rise in number counts at fainter flux densities, one could start to detect very faint background sources that are not in fact associated. Our observations, being sensitive only down to $S_{860} \approx 6 \mathrm{mJy}$, would not suffer from this problem, but, for example, Lambas et al. (2012) defined multiples by their flux density ratio, and pairs with brightest to second-brightest ratios less than 3 were considered multiples, since this value provides a reasonable cut-off for finding single dish sources whose flux densities have been seriously affected. Our observations are not able to detect ratios as high as 3 but we have probed the regime of ratios close to 1 , where single dish flux densities are the most seriously affected.

In the UDS field, we found that none of our 23 observed SCUBA-2 sources break up into two components, while the ALMA follow-up results of Simpson et al. (2015) reported 18 single dish sources breaking up into multiple components in 30 observations, a fraction of $0.60 \pm 0.14$, where the uncertainty is calculated as the square root of the number of multiples divided by the sample size. It is hard to directly compare these two results for several reasons. First, Simpson et al. (2015) targeted most of the $S_{850}>10$ mJy sources in this field, while we have followed-up those in the fainter 8-10 mJy regime, and we might expect brighter SCUBA-2 sources to have a higher chance of being composed of multiple galaxies. Second, the typical rms level obtained in the ALMA images was about $0.2 \mathrm{mJy}$, compared to our SMA images, which have about $1.5 \mathrm{mJy}$ of noise. For example, in cases where a single bright SCUBA-2 source is composed of one bright galaxy and several fainter $(\lesssim 6 \mathrm{mJy})$ galaxies below the detection limit of the SMA, we might expect to see one detection with the SMA and multiple detections with ALMA. Third, the synthesized beam of the ALMA pointings was about 0.35 arcsec, much smaller than the 2.4 arcsec synthesized beam we achieved with the SMA. We could therefore still be blending sources together, but only if they are genuinely close on the sky - Simpson et al. (2015) found only two cases with two galaxies separated by less than the SMA beamsize: UDS156.0 and UDS156.1; and UDS168.0 and UDS168.1.

We can try to estimate what these ALMA observations would have seen had they been done instead using the SMA. First, UDS156.0 and UDS156.1 would have been seen as one source as their separation is less than the average synthesized SMA beam. Next, UDS57.0 and UDS57.1 would both have been detected as a multiple since they have $870 \mu \mathrm{m}$ ALMA flux densities greater than our threshold of $6 \mathrm{mJy}$ and a separation greater than 2.4 arcsec. The remaining observations in the data set of Simpson et al. (2015) would have been single sources in our SMA observations as they all have only one galaxy above $6 \mathrm{mJy}$ as seen by ALMA at $870 \mu \mathrm{m}$. There are two exceptions: UDS286.0, UDS286.1, UDS286.2 and UDS286.3, as well as UDS199.0 and UDS199.1, would have been blank in our SMA observations as each member falls below our sensitivity. To summarize, we can speculate that had we followed up each of the 16 sources observed by 

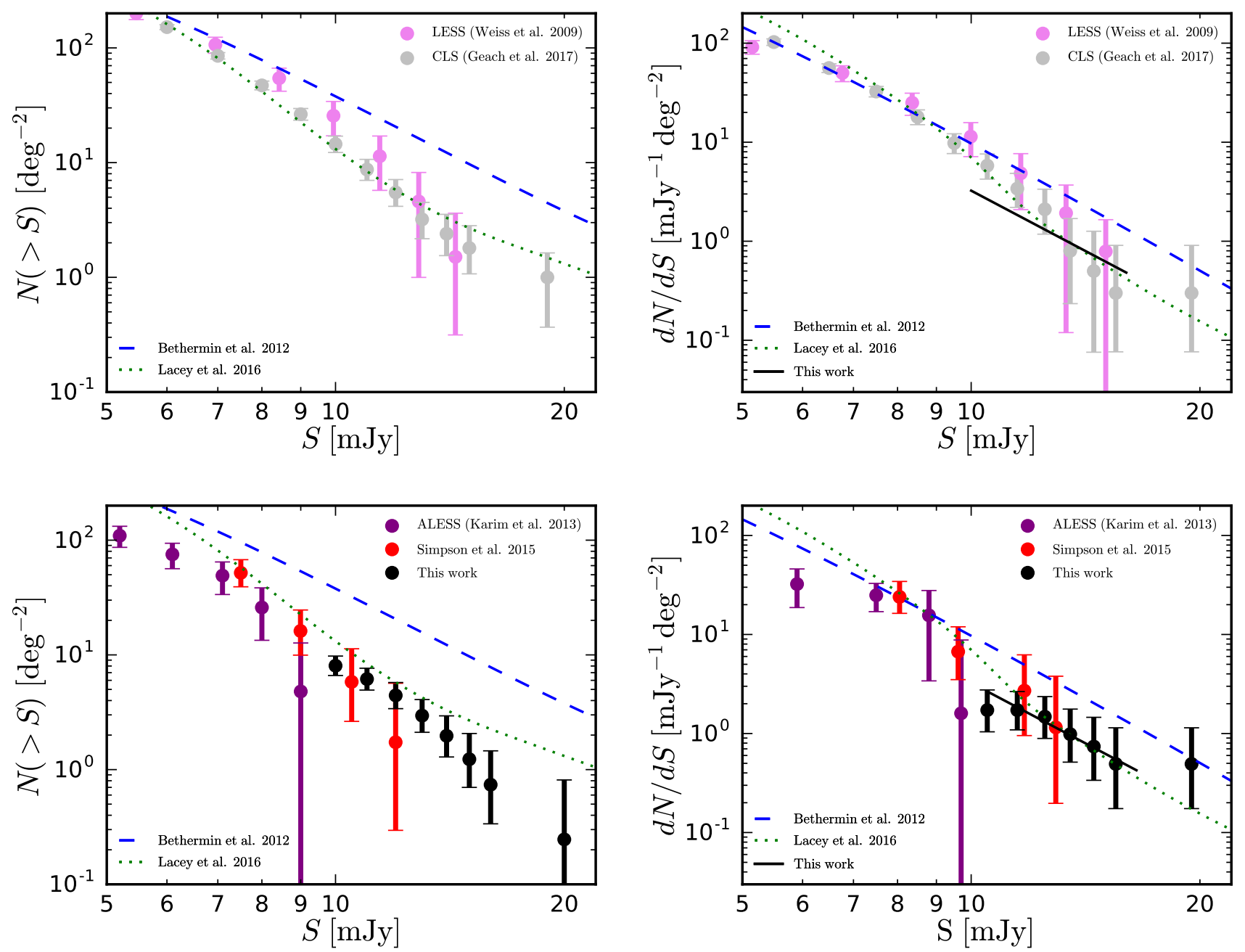

Figure 9. Cumulative and differential number counts for the two large single dish submm surveys LESS (Weiß et al. 2009) and CLS (Geach et al. 2017) on the top row. On the bottom row we show cumulative and differential number counts from Karim et al. (2013) and Simpson et al. (2015), interferometric follow-up studies of the LESS and CLS surveys, respectfully, shown along with our SMA results and the shaded region indicating where our data is no longer 100 per cent complete. Also shown are the models of Béthermin et al. (2012) and Lacey et al. (2016). The black solid line shows the best-fit power to our differential distribution between 11 and $16 \mathrm{mJy}$.

Simpson et al. (2015) used in our study with the SMA, we would have found only one pair of galaxies and two blank maps, which is a multiplicity fraction of $0.06 \pm 0.06$.

In the COSMOS field we found that one SCUBA-2 source resolved into two components. Including AzTEC11 from Younger et al. (2009), also a multiple, we obtain a multiplicity fraction of $0.07 \pm 0.05$ out of 27 SMA observations. We note that the full catalogues published in Younger et al. (2007, 2009) contained two multiples, one of which is not included in our observations since its SCUBA-2 counterpart was not detected in the CLS.

In the LHN field we found that, of the 18 SCUBA-2 sources followed-up, two break up into two galaxies, which is a multiplicity fraction of $0.11 \pm 0.08$. This field represents the highest such fraction in our sample; however, it still does not approach the multiplicity fraction seen in the Simpson et al. (2015) sample. The remaining fields, SSA22 and EGS, did not show any multiple-galaxy SCUBA-2 sources.

The fact that we did not robustly detect any sources in 13 of our pointings may in several cases be attributed to faint multiples being missed due to the noise level in our SMA observations. Using more sensitive ALMA observations, Simpson et al. (2015) found two cases of bright, $7-11$ mJy SCUBA-2 $850 \mu \mathrm{m}$ sources resolving into multiple $<6$ mJy sources at $870 \mu \mathrm{m}$, which would not be detected in most of our SMA pointings. It is thus plausible to attribute some of our null-detections to cases where the SCUBA-2 blended source is composed of multiple faint sources that are lost in the noise; however, we must be careful with this interpretation as there also are instances where the flux density threshold in our SMA maps is greater than the flux density of the SCUBA-2 source we are trying to detect. In these cases we cannot claim evidence for detecting multiplicity. Specifically, UDS14, UDS15, SSA22-03, COSMOS06, COSMOS17, LHN11 and LHN12 each have SMA flux density limits less than their observed SCUBA-2 counterpart flux densities, and so may be composed of multiple galaxies below our $4 \sigma$ limit, whereas for SSA22-04, SSA22-07, SSA22- 
09, COSMOS22, COSMOS23 and COSMOS25 we are not able to say anything about the galaxies contributing to the SCUBA-2 flux density. Under the interpretation that undetected sources constrained by our SMA observations to be fainter than their SCUBA-2 measurements are in fact multiples, we actually have observed seven more of these systems.

This interpretation would change the multiplicity fraction in the COSMOS field to $0.15 \pm 0.07$, and the multiplicity fraction in the LHN field to $0.22 \pm 0.11$, while in the SSA22 field we can calculate a fraction of $0.11 \pm 0.11$. In the UDS field, in order to properly incorporate the ALMA observations (taking into account the difference in sensitivity compared to the SMA) we should include not just UDS57.0 and UDS57.1 as a multiple but also UDS156.0 and UDS156.1, since we know that in truth the latter is a system of two bright, close galaxies, and also the two maps that would have been blank if observed with the SMA, as discussed above. Then, incorporating the two non-detections UDS14 and UDS15 from our SMA observations, we find a multiplicity fraction of $0.14 \pm 0.06$, which agrees well with the other fields.

Considering the multiplicity of our entire catalogue, we have a total of 15 multiples in 102 observations (removing the gravitational lens Orochi), which results in a multiplicity fraction of $0.15 \pm 0.04$. It is worth noting once more the assumptions we have made to get to this number. First, we have assumed that undetected sources with flux density constraints less than what was observed by SCUBA-2 are multiples, but this might not be true if an intrinsically faint SCUBA-2 source were to lie on a rather large positive noise peak. Second, we have assumed that there are no multiples where we have not been able to gain any interferometric information due to large amounts of noise, which might not be true in particular for SSA22-04, a 10 mJy SCUBA-2 $850 \mu \mathrm{m}$ source which could potentially be resolved into two similarly bright galaxies detectable by the SMA.

Our data therefore suggest that about 15 per cent of single dish submm sources brighter than $10 \mathrm{mJy}$ will be seen as multiple SMGs with similar flux densities when viewed with an angular resolution of 2.4 arcsec. This multiplicity fraction can be thought of as depending on three parameters: the minimum flux densities targeted, the sensitivity of the interferometric observations, and the angular resolution of the interferometric observations. In targeting brighter sources, we expect to observe more multiples (when strong lensing is not considered), and in addition, more sensitive instruments with better angular resolution, like ALMA, should also observe more multiples, as observed by Karim et al. (2013) and Simpson et al. (2015).

The question of wheather the multiplicity seen in our SMA images correspond directly to galaxy mergers is difficult to address with our data. First, we note that the physical scale being probed by the SMA's resolution, namely 2.4 arcsec, at a fiducial redshift of $z=2$ is about $20 \mathrm{kpc}$, which is around the same separation seen with major-mergers in the local Universe (e.g. Lambas et al. 2012, who examined a set of about 2000 galaxy pairs at $z<0.1$ ). On the other hand, with enough sensitivity one will always detect faint multiples; it has been suggested that line-of-sight projections could account for a significant fraction of the multiplicity seen in bright SMGs (Cowley et al. 2015). But the fact that we hardly see any multiples with several bright components may be useful for detecting galaxy clusters in formation. Under the assumption that these multiples are in fact physically associated galaxies, one could reasonably expect that the few instances of multiplicity where both galaxies are equally bright are massive galaxy cluster cores in the midst of formation, being such rare events. However, the sensitivity of our SMA observations only allows us to detect up to two galaxies per single SCUBA-2 source (there is no way to divide a $12 \mathrm{mJy}$ source into three parts brighter than $6 \mathrm{mJy}$, the typical sensitivity limit of our data - the two faint companions to LHN09 technically fall below our S/N threshold of 4), which may be too few to be the progenitor of today's massive galaxy clusters. Nonetheless, the fact that we do not observe very many equally bright pairs may help future work constrain massive cluster formation.

\subsection{Density of extremely luminous galaxies}

Our sample of galaxies represent some of the most luminous and intensely star-forming sites in the Universe. In order to estimate the average luminosity and SFR of our sample of galaxies, we take the set of 52 (rest frame) SEDs provided by Danielson et al. (2017) from galaxies in the ALESS and calculate their mean to derive an average SMG SED. We then fix the redshift at $z=2$ and set the normalization to get $10 \mathrm{mJy}$ at $860 \mu \mathrm{m}$, and integrate this model from 8 to $1000 \mu \mathrm{m}$ (which is the definition of the IR luminosity). This calculation results in $4.5 \times 10^{12} \mathrm{~L}_{\odot}$, and this can be converted to a SFR using the relationship from Kennicutt (1998) modified for a Chabrier IMF (Chabrier 2003) (i.e. $\left.\mathrm{SFR}\left[\mathrm{M}_{\odot} \mathrm{yr}^{-1}\right]=9.5 \times 10^{-11} \mathrm{~L}_{\mathrm{IR}} / \mathrm{L}_{\odot}\right)$ to yield $\sim 400 \mathrm{M}_{\odot} \mathrm{yr}^{-1}$.

Using the above result we can recast our number counts in terms of intrinsic SFRs. Since we see a surface density of galaxies brighter than $10 \mathrm{mJy}$ of $8_{-1}^{+2} \mathrm{deg}^{-2}$, this is a good approximation for the number of galaxies with SFRs $\gtrsim 400 \mathrm{M}_{\odot} \mathrm{yr}^{-1}$. Here we are assuming that none of our sources are being gravitationally lensed, which would reduce their intrinsic SFRs; we will address the fraction of gravitaionaly lensed galaxies in our sample in future work. Assuming half of our sources lie between $z=2-3$ and using the cosmological parameters from Planck Collaboration XIII (2016), this implies a likely volume density of $\sim 3_{-0.6}^{+0.7} \times 10^{-7} \mathrm{Mpc}^{-3}$.

The lifetimes of starbursts in SMGs are expected to be of order $100 \mathrm{Myr}$ (e.g., Swinbank et al. 2006; Tacconi et al. 2008; Hainline et al. 2011; Hickox et al. 2012). Between $z=3$ and $z=2$ the lookback time is approximately $1 \mathrm{Gyr}$, which implies that the volume density galaxies descended from this population is larger by a factor of 10 , or $\sim 3 \times 10^{-6} \mathrm{Mpc}^{-3}$. This number density can be compared to the number density of local $(z<0.1)$, red quiescent galaxies, which are the expected descendants. The local volume density as a function of stellar mass (i.e. the stellar mass function) of quiescent galaxies has been measured by selecting 'red sequence' galaxies based on their colour (Bell et al. 2003; Baldry et al. 2012) and by selecting 'star-forming sequence' galaxies based on their location on a SFR-stellar mass diagram (Moustakas et al. 2013), and both techniques agree well for stellar masses $\gtrsim 10^{11} \mathrm{M}_{\odot}$. We have taken the stellar mass function of Moustakas et al. (2013), which probed the largest stellar masses out to $10^{12} \mathrm{M}_{\odot}$ and is therefore a good comparison to our study of the most extreme galaxies, and 
have calculated the cumulative volume density as a function of stellar mass by integrating the stellar mass function. From this we find that local quiescent galaxies with stellar masses $\gtrsim 4 \times 10^{11} \mathrm{M}_{\odot}$ have the same volume density as the remnants of $\gtrsim 400 \mathrm{M}_{\odot} \mathrm{yr}^{-1}$ galaxies in our sample. We note that the above calculation is not significantly affected by any of the assumptions we made as the stellar mass function is very steep above $10^{11} \mathrm{M}_{\odot}$, changing by only about 0.5 dex over 2 orders of magnitude in volume density. Adopting a fiducial values of $500 \mathrm{M}_{\odot} \mathrm{yr}^{-1}$ for the typical SFR in our sample and assuming the bursts are constant over the $100 \mathrm{Myr}$ period, this implies a stellar mass of $5 \times 10^{10} \mathrm{M}_{\odot}$ was created during the bursts, a fraction of approximately 10 per cent the total stellar mass assembled by $z=0$.

\section{SUMMARY AND CONCLUSIONS}

Using the SMA we have followed-up 75 of the brightest SCUBA-2 CLS sources spread across $4 \mathrm{deg}^{2}$ in five fields. We have also included in our analysis 28 archival SMA and ALMA observations of similar nature to bring our total sample size to 103 single-dish submm sources. The synthesized beam of our observations was on average 2.4 arcsec FWHM and the noise $1.5 \mathrm{mJy}$ as calculated from the primary beamcorrected images, sufficient to resolve the dominant SMGs contributing to the flux density peaks seen by the SCUBA-2 instrument. Altogether, we detected 65 SMGs above $4 \sigma$, and saw three examples of a single SCUBA-2 peak breaking up into two or more bright SMGs. We also found that 12 of our pointings did not detect any SMGs, which may result from a SCUBA-2 peak breaking up into two or more SMGs fainter than our $4 \sigma$ detection limit, which is on average $6 \mathrm{mJy}$.

We simulated SCUBA-2 maps and SMA follow-up pointings using the same selection criteria as for our observations in order to estimate and correct for flux boosting in our measurements. Upon applying these corrections, we found that the posterior probability distributions of two sources peaked at $0 \mathrm{mJy}$, so we can only constrain 68 per cent upper limits on their flux densities. We tested our positional accuracy by calculating the radial distance from the peak flux density positions in our SMA images to those in the CLS maps, finding the spread to be consistent with the expected spread given the $\mathrm{S} / \mathrm{N}$ values. We also compared our deboosted flux density measurements to the deboosted flux density measurements published in the CLS, and found the median ratio to be $S_{\mathrm{SMA}} / S_{\mathrm{S} 2}=0.93 \pm 0.03$.

Assessing completeness, our sample consists of 95 per cent of the sources with $S>10 \mathrm{mJy}$ with respect to the reference fields in the CLS, and we calculate the number counts for this regime. We compare our number counts to what was found in our parent sample, finding general agreement; however, our cumulative number count is systematically lower than the parent SCUBA-2 cumulative count by $24 \pm 6$ per cent between 11 and $15 \mathrm{mJy}$. We also compare our counts to those from Simpson et al. (2015), who followed-up most of the bright sources in the UDS field of the CLS with ALMA, and we show that the two estimations are in agreement.

While multiplicity is evidently not uncommon in most of the bright single-dish sources, the effects appear not to severely affect the bright end of the number counts. We estimate an upper limit of 15 per cent for the fraction of sin- gle dish submm sources brighter than approximately $10 \mathrm{mJy}$ that resolve into two or more galaxies with similar flux densities. Instead, the most common situation involves bright single dish submm sources resolving into one slightly less bright SMG and several much fainter ones with much larger flux density ratios, which only slightly lowers previous estimates of the number of bright SMGs.

Lastly we calculate the surface density of galaxies with SFRs greater than approximately $400 \mathrm{M}_{\odot} \mathrm{yr}^{-1}$ to be $8_{-1}^{+2} \mathrm{deg}^{-2}$. Assuming half of the redshifts are between $z=2-3$, this corresponds to a volume density of $\sim 3_{-0.6}^{+0.7} \times 10^{-7} \mathrm{Mpc}^{-3}$. Taking the typical lifetimes for starbursts to be of order $100 \mathrm{Myr}$ and noting that $z=2-3$ corresponds to a lookback time of about $1 \mathrm{Gyr}$, we find a volume density of remnants to be $\sim 3 \times 10^{-6} \mathrm{Mpc}^{-3}$, which corresponds to the local volume density of quiescent galaxies with stellar masses $\gtrsim 4 \times 10^{11} \mathrm{M}_{\odot}$. Since local quiescent galaxies are expected to be descendants of the starbursting galaxies in our sample, we estimate that about 10 per cent of their total stellar mass assembled by $z=0$ was formed during these short bursts of star-formation.

\section{ACKNOWLEDGEMENTS}

The James Clerk Maxwell Telescope is now operated by the East Asian Observatory on behalf of The National Astronomical Observatory of Japan, Academia Sinica Institute of Astronomy and Astrophysics, the Korea Astronomy and Space Science Institute, the National Astronomical Observatories of China and the Chinese Academy of Sciences (Grant No. XDB09000000), with additional funding support from the Science and Technology Facilities Council of the United Kingdom and participating universities in the United Kingdom and Canada. The Submillimeter Array is a joint project between the Smithsonian Astrophysical Observatory and the Academia Sinica Institute of Astronomy and Astrophysics and is funded by the Smithsonian Institution and the Academia Sinica. The authors wish to recognize and acknowledge the very significant cultural role and reverence that the summit of Maunakea has always had within the indigenous Hawaiian community. We are most fortunate to have the opportunity to conduct observations from this mountain. This work was supported by the Natural Sciences and Research Council of Canada. IRS and AMS acknowledge support from STFC (ST/P000541/1). IRS also acknowledges the ERC Advanced Investigator programme DUSTYGAL 321334 and a Royal Society/Wolfson Merit Award. M.J.M. acknowledges the support of the National Science Centre, Poland through the POLONEZ grant 2015/19/P/ST9/04010. This project has received funding from the European Union's Horizon 2020 research and innovation programme under the Marie Skłodowska-Curie grant agreement No. 665778.

\section{REFERENCES}

Baldry I. K., et al., 2012, MNRAS, 421, 621

Barger A. J., Cowie L. L., Sanders D. B., Fulton E., Taniguchi Y., Sato Y., Kawara K., Okuda H., 1998, Nature, 394, 248

Barger A. J., Wang W.-H., Cowie L. L., Owen F. N., Chen C.-C., Williams J. P., 2012, ApJ, 761, 89 
Bell E. F., McIntosh D. H., Katz N., Weinberg M. D., 2003, ApJS, 149, 289

Bertoldi F., et al., 2007, ApJS, 172, 132

Béthermin M., et al., 2012, ApJ, 757, L23

Biggs A. D., et al., 2011, MNRAS, 413, 2314

Blain A. W., Smail I., Ivison R. J., Kneib J.-P., 1999, MNRAS, 302, 632

Blain A. W., Smail I., Ivison R. J., Kneib J.-P., Frayer D. T., 2002, Phys. Rep., 369, 111

Borys C., Scott D., Chapman S., Halpern M., Nandra K., Pope A., 2004, MNRAS, 355, 485

Casey C. M., et al., 2013, MNRAS, 436, 1919

Chabrier G., 2003, PASP, 115, 763

Chapin E. L., et al., 2009, MNRAS, 398, 1793

Chapin E. L., et al., 2011, MNRAS, 411, 505

Chapman S. C., Richards E. A., Lewis G. F., Wilson G., Barger A. J., 2001, ApJ, 548, L147

Chapman S. C., Lewis G. F., Scott D., Borys C., Richards E., 2002, ApJ, 570, 557

Chapman S. C., et al., 2003, ApJ, 585, 57

Chapman S. C., Smail I., Windhorst R., Muxlow T., Ivison R. J., 2004, ApJ, 611, 732

Chapman S. C., Blain A. W., Smail I., Ivison R. J., 2005, ApJ, 622,772

Chen C.-C., et al., 2015, ApJ, 799, 194

Condon J. J., 1992, ARA\&A, 30, 575

Coppin K., Halpern M., Scott D., Borys C., Chapman S., 2005, MNRAS, 357, 1022

Coppin K., et al., 2006, MNRAS, 372, 1621

Cowley W. I., Lacey C. G., Baugh C. M., Cole S., 2015, MNRAS, 446,1784

Danielson A. L. R., et al., 2017, ApJ, 840, 78

Dannerbauer H., Lehnert M. D., Lutz D., Tacconi L., Bertoldi F., Carilli C., Genzel R., Menten K. M., 2004, ApJ, 606, 664

Davé R., Finlator K., Oppenheimer B. D., Fardal M., Katz N., Kereš D., Weinberg D. H., 2010, MNRAS, 404, 1355

Engel H., et al., 2010, ApJ, 724, 233

Geach J. E., et al., 2017, MNRAS, 465, 1789

Gehrels N., 1986, ApJ, 303, 336

Genzel R., Baker A. J., Tacconi L. J., Lutz D., Cox P., Guilloteau S., Omont A., 2003, ApJ, 584, 633

Greve T. R., Ivison R. J., Bertoldi F., Stevens J. A., Dunlop J. S., Lutz D., Carilli C. L., 2004, MNRAS, 354, 779

Griffin M. J., et al., 2010, A\&A, 518, L3

Gruppioni C., et al., 2013, MNRAS, 432, 23

Guilloteau S., et al., 1992, A\&A, 262, 624

Hainline L. J., Blain A. W., Smail I., Alexander D. M., Armus L., Chapman S. C., Ivison R. J., 2011, ApJ, 740, 96

Hickox R. C., et al., 2012, MNRAS, 421, 284

Ho P. T. P., Moran J. M., Lo K. Y., 2004, ApJ, 616, L1

Hodge J. A., et al., 2013, ApJ, 768, 91

Holland W. S., et al., 1999, MNRAS, 303, 659

Holland W. S., et al., 2013, MNRAS, 430, 2513

Hughes D. H., et al., 1998, Nature, 394, 241

Ikarashi S., et al., 2011, MNRAS, 415, 3081

Indebetouw R., et al., 2014, ApJ, 782, L2

Iono D., et al., 2006, ApJ, 640, L1

Ivison R. J., Smail I., Le Borgne J.-F., Blain A. W., Kneib J.-P., Bezecourt J., Kerr T. H., Davies J. K., 1998, MNRAS, 298, 583

Ivison R. J., Smail I., Barger A. J., Kneib J.-P., Blain A. W., Owen F. N., Kerr T. H., Cowie L. L., 2000, MNRAS, 315, 209

Ivison R. J., et al., 2002, MNRAS, 337, 1

Ivison R. J., et al., 2007, MNRAS, 380, 199

Ivison R. J., et al., 2010, A\&A, 518, L31

Karim A., et al., 2013, MNRAS, 432, 2

Kennicutt Jr. R. C., 1998, ARA\&A, 36, 189
Koprowski M. P., Dunlop J. S., Michałowski M. J., Cirasuolo M., Bowler R. A. A., 2014, MNRAS, 444, 117

Koprowski M. P., et al., 2016, MNRAS, 458, 4321

Koprowski M. P., Dunlop J. S., Michałowski M. J., Coppin K. E. K., Geach J. E., McLure R. J., Scott D., van der Werf P. P., 2017, preprint, (arXiv:1706.00426)

Lacey C. G., et al., 2016, MNRAS, 462, 3854

Lambas D. G., Alonso S., Mesa V., O'Mill A. L., 2012, A\&A, 539, A45

Lilly S. J., Eales S. A., Gear W. K. P., Hammer F., Le Fèvre O., Crampton D., Bond J. R., Dunne L., 1999, ApJ, 518, 641

MacKenzie T. P., et al., 2017, MNRAS, 468, 4006

Magnelli B., et al., 2012, A\&A, 539, A155

Magnelli B., et al., 2013, A\&A, 553, A132

Magnelli B., et al., 2015, A\&A, 573, A45

Michałowski M. J., et al., 2017, MNRAS, 469, 492

Miettinen O., et al., 2015, A\&A, 584, A32

Moustakas J., et al., 2013, ApJ, 767, 50

Narayanan D., et al., 2015, Nature, 525, 496

Oliver S. J., et al., 2010, A\&A, 518, L21

Pascale E., et al., 2008, ApJ, 681, 400

Planck Collaboration XIII 2016, A\&A, 594, A13

Pope A., et al., 2006, MNRAS, 370, 1185

Riechers D. A., et al., 2013, Nature, 496, 329

Scott S. E., et al., 2002, MNRAS, 331, 817

Scott K. S., et al., 2008, MNRAS, 385, 2225

Simpson J. M., et al., 2014, ApJ, 788, 125

Simpson J. M., et al., 2015, ApJ, 799, 81

Simpson J. M., et al., 2017, ApJ, 839, 58

Siringo G., et al., 2009, A\&A, 497, 945

Smail I., Ivison R. J., Blain A. W., 1997, ApJ, 490, L5

Smail I., Ivison R. J., Owen F. N., Blain A. W., Kneib J.-P., 2000, ApJ, 528, 612

Smolčić V., et al., 2012a, ApJS, 200, 10

Smolčić V., et al., 2012b, A\&A, 548, A4

Swinbank A. M., Chapman S. C., Smail I., Lindner C., Borys C., Blain A. W., Ivison R. J., Lewis G. F., 2006, MNRAS, 371, 465

Swinbank A. M., et al., 2014, MNRAS, 438, 1267

Tacconi L. J., et al., 2008, ApJ, 680, 246

Targett T. A., et al., 2013, MNRAS, 432, 2012

Toft S., et al., 2014, ApJ, 782, 68

Valiante E., et al., 2016, MNRAS, 462, 3146

Wang W.-H., Cowie L. L., Barger A. J., 2004, ApJ, 613, 655

Wang W.-H., Cowie L. L., Barger A. J., Williams J. P., 2011, ApJ, 726, L18

Wardlow J. L., et al., 2011, MNRAS, 415, 1479

Weiß A., et al., 2009, ApJ, 707, 1201

Wilson G. W., et al., 2008, MNRAS, 386, 807

Wootten A., Thompson A. R., 2009, IEEE Proceedings, 97, 1463

Younger J. D., et al., 2007, ApJ, 671, 1531

Younger J. D., et al., 2009, ApJ, 704, 803

Yun M. S., Reddy N. A., Condon J. J., 2001, ApJ, 554, 803

Yun M. S., et al., 2012, MNRAS, 420, 957

van Dokkum P. G., et al., 2015, ApJ, 813, 23

\section{APPENDIX A: DATA TABLES}

Here we provide data tables detailing our interferometric sample. Each of the five fields used in our study are summarized in a single table and ordered by decreasing deboosted SCUBA-2 flux density. The columns give the source name, the SCUBA-2 position, the SMA (or ALMA) position, the SCUBA-2 observed flux density, the deboosted SCUBA-2 flux density, the SMA (or ALMA) observed flux density and the SMA deboosted flux density. For SMA pointings that 
did not detect any galaxies above $4 \sigma$ we provide flux density upper limits. For sources that were deboosted to $0 \mathrm{mJy}$, we also provide $4 \sigma$ upper limits. All sources are sorted by their deboosted SCUBA-2 flux density. We have used ALMA data from Simpson et al. (2015) for some of the sources in the UDS field; these sources are marked with $\mathrm{a}^{\mathrm{b}}$. We have also used SMA data from Younger et al. (2007) and Younger et al. (2009) for some of the sources in the COSMOS field; these sources are marked with $\mathrm{a}^{\mathrm{c}}$ and $\mathrm{a}^{\mathrm{d}}$, respectively.

This paper has been typeset from a $\mathrm{T}_{\mathrm{E}} \mathrm{X} / \mathrm{LAT}_{\mathrm{E}} \mathrm{X}$ file prepared by the author. 
Table A1. SMA sample plus archival ALMA data for the UDS field, ordered by decreasing deboosted SCUBA-2 flux density. Sources

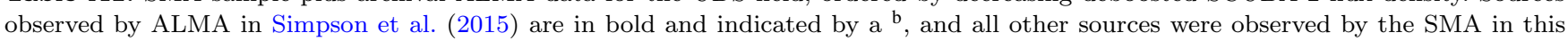
work.

\begin{tabular}{|c|c|c|c|c|c|c|}
\hline Source & $\begin{array}{c}\text { RA/Dec SCUBA-2 } \\
(\mathrm{J} 2000)\end{array}$ & $\begin{array}{c}\text { RA/Dec SMA (ALMA) } \\
\text { (J2000) }\end{array}$ & $S_{\mathrm{S} 2}^{\mathrm{obs}}[\mathrm{mJy}]$ & $S_{\mathrm{S} 2}[\mathrm{mJy}]$ & $\begin{array}{l}S_{\mathrm{SMA}}^{\mathrm{obs}}[\mathrm{mJy}] \\
\left(S_{\mathrm{ALMA}}^{\mathrm{Obs}}\right)\end{array}$ & $\begin{array}{l}S_{\mathrm{SMA}}[\mathrm{mJy}] \\
\left(S_{\mathrm{ALMA}}\right)\end{array}$ \\
\hline Orochi $^{\mathrm{a}}$ & $02: 18: 30.77-05: 31: 30.8$ & $02: 18: 30.68-05: 31: 31.7$ & $52.7 \pm 0.9$ & $52.7 \pm 1.2$ & $90.7 \pm 20.7$ & \\
\hline UDS156.0 ${ }^{\mathrm{b}}$ & $02: 18: 24.33-05: 22: 56.8$ & $02: 18: 24.14-05: 22: 55.3$ & $16.7 \pm 0.9$ & $16.4 \pm 1.3$ & $9.7 \pm 0.7$ & $9.7 \pm 0.7$ \\
\hline $156.1^{\mathrm{b}}$ & $02: 18: 24.33-05: 22: 56.8$ & $02: 18: 24.24-05: 22: 56.9$ & $16.7 \pm 0.9$ & $16.4 \pm 1.3$ & $8.5 \pm 0.7$ & $8.5 \pm 0.7$ \\
\hline UDS57.0 ${ }^{\mathrm{b}}$ & $02: 19: 21.19-04: 56: 52.5$ & $02: 19: 21.14-04: 56: 51.3$ & $13.0 \pm 0.9$ & $12.8 \pm 1.7$ & $9.5 \pm 0.6$ & $9.5 \pm 0.6$ \\
\hline $57.1^{\mathrm{b}}$ & $02: 19: 21.19-04: 56: 52.5$ & $02: 19: 20.88-04: 56: 52.9$ & $13.0 \pm 0.9$ & $12.8 \pm 1.7$ & $6.0 \pm 0.9$ & $5.8 \pm 0.9$ \\
\hline $57.2^{\mathrm{b}}$ & $02: 19: 21.19-04: 56: 52.5$ & $02: 19: 21.41-04: 56: 49.0$ & $13.0 \pm 0.9$ & $12.8 \pm 1.7$ & $1.8 \pm 0.6$ & $1.5 \pm 0.6$ \\
\hline $\mathbf{5 7 . 3} \mathbf{3}^{\mathrm{b}}$ & $02: 19: 21.19-04: 56: 52.5$ & $02: 19: 21.39-04: 56: 38.8$ & $13.0 \pm 0.9$ & $12.8 \pm 1.7$ & $2.7 \pm 1.0$ & $2.1 \pm 1.0$ \\
\hline UDS03 & $02: 15: 55.41-05: 24: 56.2$ & $02: 15: 55.10-05: 24: 56.6$ & $12.8 \pm 1.3$ & $12.0 \pm 1.8$ & $13.7 \pm 1.4$ & $13.1_{-15}^{+1.2}$ \\
\hline UDS361. $0^{\mathrm{b}}$ & $02: 16: 48.08-05: 01: 30.7$ & $02: 16: 47.92-05: 01: 29.8$ & $11.5 \pm 0.9$ & $11.3 \pm 1.7$ & $11.8 \pm 0.6$ & $11.8^{-1.5} \pm 0.6$ \\
\hline $361.1^{\mathrm{b}}$ & $02: 16: 48.08-05: 01: 30.7$ & $02: 16: 47.73-05: 01: 25.8$ & $11.5 \pm 0.9$ & $11.3 \pm 1.7$ & $2.6 \pm 0.7$ & $2.0 \pm 0.7$ \\
\hline UDS286.0 $^{\mathrm{b}}$ & $02: 17: 25.81-05: 25: 36.9$ & $02: 17: 25.73-05: 25: 41.2$ & $11.4 \pm 0.9$ & $11.2 \pm 1.7$ & $5.2 \pm 0.7$ & $5.1 \pm 0.7$ \\
\hline $286.1^{\text {b }}$ & $02: 17: 25.81-05: 25: 36.9$ & $02: 17: 25.63-05: 25: 33.7$ & $11.4 \pm 0.9$ & $11.2 \pm 1.7$ & $5.1 \pm 0.6$ & $5.0 \pm 0.6$ \\
\hline $286.2^{\mathrm{b}}$ & $02: 17: 25.81-05: 25: 36.9$ & $02: 17: 25.80-05: 25: 37.5$ & $11.4 \pm 0.9$ & $11.2 \pm 1.7$ & $2.7 \pm 0.6$ & $2.6 \pm 0.6$ \\
\hline $286.3^{\mathrm{b}}$ & $02: 17: 25.81-05: 25: 36.9$ & $02: 17: 25.52-05: 25: 36.7$ & $11.4 \pm 0.9$ & $11.2 \pm 1.7$ & $1.7 \pm 0.6$ & $1.4 \pm 0.6$ \\
\hline UDS269.0 ${ }^{\mathrm{b}}$ & $02: 17: 30.50-05: 19: 22.9$ & $02: 17: 30.44-05: 19: 22.4$ & $11.0 \pm 0.9$ & $10.7 \pm 1.4$ & $12.9 \pm 0.6$ & $12.9 \pm 0.6$ \\
\hline $269.1^{\text {b }}$ & $02: 17: 30.50-05: 19: 22.9$ & $02: 17: 30.25-05: 19: 18.4$ & $11.0 \pm 0.9$ & $10.7 \pm 1.4$ & $2.6 \pm 0.7$ & $2.1 \pm 0.7$ \\
\hline UDS08 & $02: 15: 56.03-04: 55: 10.3$ & $02: 15: 55.95-04: 55: 08.6$ & $10.9 \pm 1.0$ & $10.5 \pm 1.3$ & $10.1 \pm 1.7$ & $8.9_{-16}^{+1.6}$ \\
\hline UDS204. $0^{\mathrm{b}}$ & $02: 18: 03.04-05: 28: 42.9$ & $02: 18: 03.01-05: 28: 41.9$ & $10.7 \pm 0.9$ & $10.4 \pm 1.2$ & $11.6 \pm 0.6$ & $11.6^{-1.6} 0.6$ \\
\hline $204.1^{\mathrm{b}}$ & $02: 18: 03.04-05: 28: 42.9$ & $02: 18: 03.01-05: 28: 32.5$ & $10.7 \pm 0.9$ & $10.4 \pm 1.2$ & $2.9 \pm 0.9$ & $2.2 \pm 0.9$ \\
\hline UDS202.0 $0^{\mathrm{b}}$ & $02: 18: 05.71-05: 10: 50.9$ & $02: 18: 05.65-05: 10: 49.6$ & $11.0 \pm 0.9$ & $10.4 \pm 1.5$ & $10.5 \pm 0.5$ & $10.5 \pm 0.5$ \\
\hline $202.1^{\mathrm{b}}$ & $02: 18: 05.71-05: 10: 50.9$ & $02: 18: 05.05-05: 10: 46.3$ & $11.0 \pm 0.9$ & $10.4 \pm 1.5$ & $3.9 \pm 0.9$ & $3.5 \pm 0.9$ \\
\hline UDS09 & $02: 17: 38.95-04: 33: 37.0$ & $02: 17: 38.82-04: 33: 34.1$ & $10.9 \pm 1.3$ & $10.1 \pm 1.2$ & $13.9 \pm 0.8$ & $13.6_{-0.7}^{+0.9}$ \\
\hline UDS11 & $02: 16: 43.77-05: 17: 54.7$ & $02: 16: 43.72-05: 17: 53.5$ & $10.1 \pm 0.9$ & $9.8 \pm 1.4$ & $10.0 \pm 1.8$ & $8.6_{-1.5}^{+1.7}$ \\
\hline UDS306.0 ${ }^{\mathrm{b}}$ & $02: 17: 17.23-05: 33: 26.8$ & $02: 17: 17.07-05: 33: 26.6$ & $9.9 \pm 1.0$ & $9.7 \pm 1.3$ & $8.3 \pm 0.5$ & $8.3 \pm 0.5$ \\
\hline $306.1^{\mathrm{b}}$ & $02: 17: 17.23-05: 33: 26.8$ & $02: 17: 17.16-05: 33: 32.5$ & $9.9 \pm 1.0$ & $9.7 \pm 1.3$ & $2.6 \pm 0.4$ & $2.3 \pm 0.4$ \\
\hline $306.2^{\mathrm{b}}$ & $02: 17: 17.23-05: 33: 26.8$ & $02: 17: 16.81-05: 33: 31.8$ & $9.9 \pm 1.0$ & $9.7 \pm 1.3$ & $3.0 \pm 0.9$ & $2.3 \pm 0.9$ \\
\hline UDS14 & $02: 16: 30.77-05: 24: 02.6$ & Undetected & $9.6 \pm 0.9$ & $9.4 \pm 1.2$ & $<6.1$ & \\
\hline UDS15 & $02: 18: 03.57-04: 55: 26.9$ & Undetected & $9.6 \pm 0.9$ & $9.4 \pm 1.3$ & $<5.1$ & \\
\hline UDS16 & $02: 19: 02.24-05: 28: 56.6$ & $02: 19: 02.05-05: 28: 56.7$ & $9.5 \pm 1.0$ & $9.3 \pm 1.4$ & $6.5 \pm 1.5$ & $6.1_{-1.6}^{+1.3}$ \\
\hline UDS18 & $02: 17: 44.29-05: 20: 08.9$ & $02: 17: 44.22-05: 20: 09.8$ & $9.3 \pm 0.9$ & $9.1 \pm 1.3$ & $8.9 \pm 1.5$ & $8.1_{-1.4}^{+1.6}$ \\
\hline UDS13 & $02: 19: 27.31-04: 45: 08.5$ & $02: 19: 27.17-04: 45: 06.1$ & $9.8 \pm 1.1$ & $9.0 \pm 1.6$ & $15.3 \pm 1.1$ & $14.9_{-1.2}^{+1.4}$ \\
\hline UDS109.0 $0^{\mathrm{b}}$ & $02: 18: 50.32-05: 27: 22.7$ & $02: 18: 50.07-05: 27: 25.5$ & $9.4 \pm 0.9$ & $9.0 \pm 1.5$ & $7.7 \pm 0.7$ & $7.6^{-1.2} 0.7$ \\
\hline $109.1^{\mathrm{b}}$ & $02: 18: 50.32-05: 27: 22.7$ & $02: 18: 50.30-05: 27: 17.2$ & $9.4 \pm 0.9$ & $9.0 \pm 1.5$ & $4.3 \pm 0.6$ & $4.2 \pm 0.6$ \\
\hline UDS48.0 $0^{\mathrm{b}}$ & $02: 19: 24.66-04: 53: 00.5$ & $02: 19: 24.57-04: 53: 00.2$ & $8.9 \pm 0.8$ & $8.9 \pm 1.3$ & $7.5 \pm 0.5$ & $7.5 \pm 0.5$ \\
\hline $48.1^{\mathrm{b}}$ & $02: 19: 24.66-04: 53: 00.5$ & $02: 19: 24.62-04: 52: 56.9$ & $8.9 \pm 0.8$ & $8.9 \pm 1.3$ & $1.6 \pm 0.5$ & $1.4 \pm 0.5$ \\
\hline UDS20 & $02: 17: 30.51-04: 59: 36.9$ & $02: 17: 30.61-04: 59: 36.8$ & $9.1 \pm 0.9$ & $8.7 \pm 1.4$ & $9.0 \pm 1.4$ & $8.2_{-1.3}^{+1.3}$ \\
\hline UDS199.0 $^{\mathrm{b}}$ & 02:18:07.31 -04:44:12.9 & 02:18:07.18 -04:44:13.8 & $9.2 \pm 0.9$ & $8.5 \pm 1.4$ & $4.3 \pm 0.6$ & $4.2 \pm 0.6$ \\
\hline $199.1^{\mathrm{b}}$ & $02: 18: 07.31-04: 44: 12.9$ & $02: 18: 07.19-04: 44: 10.9$ & $9.2 \pm 0.9$ & $8.5 \pm 1.4$ & $2.5 \pm 0.5$ & $2.4 \pm 0.5$ \\
\hline UDS22 & $02: 16: 11.81-05: 00: 54.5$ & $02: 16: 11.72-05: 00: 54.0$ & $9.0 \pm 0.8$ & $8.5 \pm 1.2$ & $15.0 \pm 1.4$ & $14.1_{-13}^{+1.5}$ \\
\hline UDS160.0 ${ }^{\mathrm{b}}$ & $02: 18: 23.79-05: 11: 40.9$ & $02: 18: 23.73-05: 11: 38.5$ & $8.8 \pm 0.9$ & $8.4 \pm 1.4$ & $7.9 \pm 0.6$ & $7.9^{-1.3} \pm 0.6$ \\
\hline UDS110.0 ${ }^{\mathrm{b}}$ & $02: 18: 48.43-05: 18: 06.7$ & $02: 18: 48.24-05: 18: 05.2$ & $8.4 \pm 0.9$ & $8.2 \pm 1.4$ & $7.7 \pm 0.6$ & $7.7 \pm 0.6$ \\
\hline $110.1^{b}$ & $02: 18: 48.43-05: 18: 06.7$ & $02: 18: 48.76-05: 18: 02.1$ & $8.4 \pm 0.9$ & $8.2 \pm 1.4$ & $2.5 \pm 0.8$ & $2.0 \pm 0.8$ \\
\hline UDS21 & $02: 19: 34.14-04: 44: 40.4$ & $02: 19: 34.15-04: 44: 38.1$ & $9.0 \pm 1.2$ & $8.2 \pm 1.5$ & $10.3 \pm 1.0$ & $9.9_{-1.0}^{+0.9}$ \\
\hline UDS337.0 ${ }^{\mathrm{b}}$ & $02: 16: 41.11-05: 03: 52.7$ & $02: 16: 41.11-05: 03: 51.4$ & $8.4 \pm 0.9$ & $8.0 \pm 1.2$ & $8.1 \pm 0.5$ & $8.1^{-1.0} \pm 0.5$ \\
\hline UDS29 & $02: 17: 39.87-05: 29: 18.9$ & $02: 17: 39.78-05: 29: 19.1$ & $8.3 \pm 0.9$ & $8.0 \pm 1.3$ & $11.6 \pm 1.1$ & $11.2_{-1.2}^{+1.0}$ \\
\hline UDS79.0 ${ }^{\mathrm{b}}$ & $02: 19: 10.09-05: 00: 08.6$ & $02: 19: 09.94-05: 00: 08.6$ & $8.1 \pm 0.9$ & $7.9 \pm 1.4$ & $7.7 \pm 0.5$ & $7.7^{-1.2} \pm 0.5$ \\
\hline UDS30 & $02: 17: 55.27-04: 47: 22.9$ & $02: 17: 55.05-04: 47: 22.9$ & $8.3 \pm 0.9$ & $7.8 \pm 1.2$ & $7.4 \pm 1.1$ & $7.1_{-1.0}^{+1.0}$ \\
\hline UDS28 & $02: 19: 42.53-05: 18: 04.3$ & $02: 19: 42.45-05: 18: 03.6$ & $8.4 \pm 1.1$ & $7.6 \pm 1.6$ & $9.0 \pm 1.0$ & $8.6_{-1.0}^{+0.0}$ \\
\hline UDS36 & $02: 17: 12.19-04: 43: 18.9$ & $02: 17: 12.21-04: 43: 16.5$ & $8.0 \pm 0.9$ & $7.6 \pm 1.2$ & $8.5 \pm 1.4$ & $7.8_{-1.2}^{+1.0}$ \\
\hline UDS34 & $02: 17: 42.15-04: 56: 28.9$ & $02: 17: 41.92-04: 56: 29.8$ & $8.0 \pm 0.9$ & $7.6 \pm 1.3$ & $7.9 \pm 1.2$ & $7.6_{-1.3}^{+1.2}$ \\
\hline UDS35 & $02: 16: 40.43-05: 13: 38.7$ & $02: 16: 40.40-05: 13: 35.9$ & $8.0 \pm 0.9$ & $7.6 \pm 1.3$ & $7.1 \pm 1.4$ & $6.6_{-1.4}^{+1.3}$ \\
\hline UDS37 & $02: 16: 38.44-05: 01: 22.7$ & $02: 16: 38.33-05: 01: 21.4$ & $7.9 \pm 0.9$ & $7.5 \pm 1.3$ & $8.4 \pm 1.3$ & $7.8_{-1.2}^{+1.4}$ \\
\hline UDS39 & $02: 16: 40.57-05: 11: 00.7$ & $02: 16: 40.59-05: 10: 58.8$ & $7.9 \pm 0.9$ & $7.5 \pm 1.4$ & $7.9 \pm 1.0$ & $7.6_{-1.0}^{+0.9}$ \\
\hline UDS40 & $02: 17: 27.43-05: 06: 44.9$ & $02: 17: 27.29-05: 06: 42.8$ & $7.8 \pm 0.9$ & $7.5 \pm 1.2$ & $6.9 \pm 1.1$ & $6.6_{-1.0}^{+1.0}$ \\
\hline UDS168.0 ${ }^{\mathrm{b}}$ & $02: 18: 20.46-05: 31: 44.8$ & $02: 18: 20.40-05: 31: 43.2$ & $8.2 \pm 0.9$ & $7.5 \pm 1.4$ & $6.7 \pm 0.6$ & $6.7^{-1.0} \pm 0.6$ \\
\hline $168.1^{\mathrm{b}}$ & $02: 18: 20.46-05: 31: 44.8$ & $02: 18: 20.31-05: 31: 41.7$ & $8.2 \pm 0.9$ & $7.5 \pm 1.4$ & $3.0 \pm 0.6$ & $2.8 \pm 0.6$ \\
\hline $168.2^{\mathrm{b}}$ & $02: 18: 20.46-05: 31: 44.8$ & $02: 18: 20.17-05: 31: 38.6$ & $8.2 \pm 0.9$ & $7.5 \pm 1.4$ & $2.0 \pm 0.7$ & $1.6 \pm 0.7$ \\
\hline UDS33 & $02: 15: 46.99-05: 18: 52.2$ & $02: 15: 46.70-05: 18: 49.2$ & $8.1 \pm 1.2$ & $7.4 \pm 1.4$ & $10.3 \pm 1.0$ & $9.9_{-10}^{+1.0}$ \\
\hline UDS218. $0^{\mathrm{b}}$ & $02: 17: 54.87-05: 23: 22.9$ & $02: 17: 54.80-05: 23: 23.0$ & $7.6 \pm 0.9$ & $7.2 \pm 1.3$ & $6.6 \pm 0.7$ & $6.6^{-1.0} \pm 0.7$ \\
\hline UDS38 & $02: 16: 46.07-05: 03: 46.7$ & $02: 16: 46.17-05: 03: 48.9$ & $7.9 \pm 0.9$ & $7.2 \pm 1.3$ & $6.9 \pm 1.6$ & $6.3_{-1.5}^{+1.5}$ \\
\hline
\end{tabular}

a From Ikarashi et al. (2011) using the SMA at $860 \mu \mathrm{m}$.

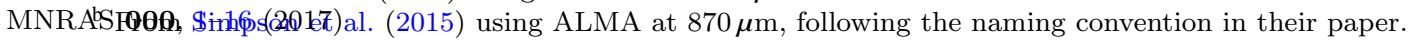


Table A2. SMA sample for the SSA22 field, ordered by decreasing deboosted SCUBA-2 flux density. All observations are from this work.

\begin{tabular}{|c|c|c|c|c|c|c|}
\hline Source & $\begin{array}{c}\text { RA/Dec SCUBA-2 } \\
(\mathrm{J} 2000)\end{array}$ & $\begin{array}{l}\text { RA/Dec SMA } \\
\quad(\mathrm{J} 2000)\end{array}$ & $S_{\mathrm{S} 2}^{\mathrm{obs}}[\mathrm{mJy}]$ & $S_{\mathrm{S} 2}[\mathrm{mJy}]$ & $S_{\mathrm{SMA}}^{\mathrm{obs}}[\mathrm{mJy}]$ & $S_{\mathrm{SMA}}[\mathrm{mJy}]$ \\
\hline SSA22-01 & $22: 17: 32.50+00: 17: 40.4$ & $22: 17: 32.43+00: 17: 44.1$ & $14.5 \pm 1.1$ & $14.5 \pm 1.4$ & $12.2 \pm 1.8$ & $10.6_{-1.8}^{+1.9}$ \\
\hline SSA22-03 & $22: 16: 56.10+00: 28: 44.4$ & Undetected & $11.1 \pm 1.2$ & $10.7 \pm 1.4$ & $<8.7$ & \\
\hline SSA22-02 & $22: 16: 59.96+00: 10: 40.4$ & $22: 16: 59.83+00: 10: 37.1$ & $10.8 \pm 1.1$ & $10.2 \pm 1.5$ & $9.3 \pm 1.6$ & $8.2_{-16}^{+1.5}$ \\
\hline SSA22-04 & $22: 16: 51.43+00: 18: 20.4$ & Undetected & $10.4 \pm 1.1$ & $10.0 \pm 1.4$ & $<12.6$ & \\
\hline SSA22-08 & $22: 18: 06.63+00: 05: 20.4$ & $22: 18: 06.60+00: 05: 20.5$ & $10.0 \pm 1.3$ & $8.8 \pm 1.8$ & $9.5 \pm 1.6$ & $8.2_{-1.3}^{+1.7}$ \\
\hline SSA22-07 & $22: 17: 18.90+00: 18: 06.4$ & Undetected & $8.5 \pm 1.1$ & $7.9 \pm 1.3$ & $<8.5$ & \\
\hline SSA22-06 & $22: 18: 06.36+00: 11: 34.4$ & $22: 18: 06.48+00: 11: 34.7$ & $8.3 \pm 1.1$ & $7.7 \pm 1.5$ & $9.9 \pm 1.3$ & $9.2^{+1.3}$ \\
\hline SSA22-05 & $22: 17: 34.10+00: 13: 52.4$ & $22: 17: 33.90+00: 13: 52.3$ & $7.9 \pm 1.1$ & $7.3 \pm 1.1$ & $11.7 \pm 2.0$ & $9.9_{-1.8}^{+2.0}$ \\
\hline SSA22-09 & $22: 17: 42.23+00: 17: 00.4$ & Undetected & $6.7 \pm 1.1$ & $6.0 \pm 1.4$ & $<8.5$ & \\
\hline
\end{tabular}

Table A3. SMA sample plus archival SMA data for the COSMOS field, ordered by decreasing deboosted SCUBA-2 flux density. Sources observed by the SMA in Younger et al. (2007) are in bold and indicated by a ${ }^{c}$, sources observed by the SMA in Younger et al. (2009) are in bold and indicated by $\mathrm{a}^{\mathrm{d}}$, and all other sources were observed by the SMA in this work. Flux density measurements from Younger et al. (2007) and Younger et al. (2009) were not deboosted. Values of N/A in the $S_{\mathrm{SMA}}$ column indicate sources where our deboosting simulation was not applicable.

\begin{tabular}{|c|c|c|c|c|c|c|}
\hline Source & $\begin{array}{c}\text { RA/Dec SCUBA-2 } \\
(\mathrm{J} 2000)\end{array}$ & $\begin{array}{l}\text { RA/Dec SMA } \\
\quad(\text { J2000) }\end{array}$ & $S_{\mathrm{S} 2}^{\mathrm{obs}}[\mathrm{mJy}]$ & $S_{\mathrm{S} 2}[\mathrm{mJy}]$ & $S_{\mathrm{SMA}}^{\mathrm{obs}}[\mathrm{mJy}]$ & $S_{\mathrm{SMA}}[\mathrm{mJy}]$ \\
\hline AzTEC1 $^{c}$ & $09: 59: 42.89+02: 29: 36.5$ & $09: 59: 42.86+02: 29: 38.2$ & $16.7 \pm 1.5$ & $16.0 \pm 3.0$ & $15.6 \pm 1.1$ & \\
\hline $\operatorname{AzTEC2}^{\mathrm{c}}$ & $10: 00: 08.11+02: 26: 12.6$ & $10: 00: 08.05+02: 26: 12.2$ & $15.4 \pm 1.4$ & $14.7 \pm 2.3$ & $12.4 \pm 1.0$ & \\
\hline COSMOS05 & $09: 59: 22.99+02: 51: 36.4$ & $09: 59: 22.99+02: 51: 36.4$ & $14.0 \pm 1.5$ & $13.0 \pm 1.7$ & $13.7 \pm 2.3$ & $11.3_{-2.2}^{+2.4}-1-10$ \\
\hline COSMOS06 & $09: 58: 42.40+02: 54: 42.2$ & Undetected & $14.0 \pm 1.5$ & $13.0 \pm 2.1$ & $<8.1$ & \\
\hline COSMOS10 & $10: 00: 15.72+02: 15: 48.6$ & $10: 00: 15.72+02: 15: 48.6$ & $12.9 \pm 0.8$ & $12.9 \pm 1.2$ & $16.8 \pm 1.5$ & $15.8_{-1.5}^{+1.7}$ \\
\hline COSMOS07 & $09: 58: 37.92+02: 14: 06.3$ & $09: 58: 37.99+02: 14: 08.5$ & $13.2 \pm 1.0$ & $12.4 \pm 1.5$ & $13.8 \pm 2.8$ & $10.7_{-3.1}^{+2.34}$ \\
\hline COSMOS09 & $10: 00: 57.22+02: 20: 12.6$ & $10: 00: 57.22+02: 20: 12.6$ & $13.0 \pm 1.5$ & $12.1 \pm 2.2$ & $12.5 \pm 2.5$ & $9.7^{+2.5}$ \\
\hline AzTEC9 $^{d}$ & $09: 59: 57.44+02: 27: 28.6$ & $09: 59: 57.25+02: 27: 30.6$ & $12.4 \pm 1.4$ & $11.8 \pm 1.9$ & $9.0 \pm 2.2$ & \\
\hline COSMOS08 & $09: 59: 10.31+02: 48: 54.4$ & $09: 59: 10.34+02: 48: 55.5$ & $13.1 \pm 1.6$ & $11.7 \pm 2.1$ & $12.7 \pm 2.0$ & $11.3_{-2.3}^{+1.6}$ \\
\hline COSMOS11a & $09: 58: 45.89+02: 43: 26.3$ & $09: 58: 45.95+02: 43: 29.1$ & $12.5 \pm 1.6$ & $11.5 \pm 2.0$ & $8.6 \pm 1.1$ & $8.0_{-1.0}^{+1.3}$ \\
\hline $11 b$ & $09: 58: 45.89+02: 43: 26.3$ & $09: 58: 46.06+02: 43: 31.5$ & $12.5 \pm 1.6$ & $11.5 \pm 2.0$ & $5.1 \pm 1.1$ & $\mathrm{~N} / \mathrm{A}$ \\
\hline COSMOS15 & $09: 57: 49.03+02: 46: 15.9$ & $09: 57: 48.93+02: 46: 19.9$ & $11.8 \pm 1.5$ & $11.2 \pm 2.1$ & $11.2 \pm 2.0$ & $9.7_{-2.2}^{+1.6}$ \\
\hline AzTEC5 $^{c}$ & $10: 00: 19.86+02: 32: 04.6$ & $10: 00: 19.75+02: 32: 04.4$ & $12.0 \pm 1.4$ & $11.2 \pm 2.2$ & $9.3 \pm 1.3$ & \\
\hline COSMOS14 & $10: 00: 13.46+01: 37: 04.7$ & $10: 00: 13.47+01: 37: 04.3$ & $12.0 \pm 1.5$ & $11.0 \pm 1.8$ & $12.2 \pm 1.2$ & $11.7_{-1.3}^{+1.0}$ \\
\hline COSMOS17 & $10: 00: 04.78+02: 30: 44.6$ & Undetected & $11.2 \pm 1.4$ & $11.0 \pm 1.8$ & $<8.4$ & \\
\hline $\operatorname{AzTEC12}^{\mathrm{d}}$ & $10: 00: 35.34+02: 43: 52.6$ & $10: 00: 35.29+02: 43: 53.4$ & $11.6 \pm 1.3$ & $10.9 \pm 2.0$ & $13.5 \pm 1.8$ & \\
\hline COSMOS18 & $09: 58: 40.46+02: 05: 14.4$ & $09: 58: 40.28+02: 05: 14.5$ & $11.1 \pm 1.5$ & $10.4 \pm 2.1$ & $10.9 \pm 1.7$ & $9.7_{-1.7}^{+1.6}$ \\
\hline AzTEC $^{\mathrm{d}}$ & $09: 59: 59.44+02: 34: 38.6$ & $09: 59: 59.34+02: 34: 41.0$ & $10.9 \pm 1.4$ & $10.1 \pm 1.8$ & $19.7 \pm 1.8$ & \\
\hline $\mathrm{AzTEC}^{\mathrm{c}}$ & $10: 00: 17.99+02: 48: 30.5$ & $10: 00: 18.06+02: 48: 30.5$ & $10.8 \pm 1.4$ & $9.7 \pm 2.0$ & $12.0 \pm 1.5$ & \\
\hline COSMOS21 & $09: 59: 07.63+02: 58: 36.3$ & $09: 59: 07.49+02: 58: 39.3$ & $10.6 \pm 1.5$ & $9.5 \pm 2.0$ & $9.9 \pm 1.9$ & $8.3_{-1.8}^{+1.8}$ \\
\hline AzTEC3 $^{c}$ & $10: 00: 20.79+02: 35: 20.6$ & $10: 00: 20.70+02: 35: 20.5$ & $9.2 \pm 1.3$ & $8.6 \pm 1.5$ & $8.7 \pm 1.5$ & \\
\hline AzTEC11. $\mathbf{N}^{\mathrm{d}}$ & 10:00:08.91 +02:40:10.6 & 10:00:08.91 +02:40:09.6 & $9.3 \pm 1.4$ & $8.3 \pm 1.8$ & $10.0 \pm 2.1$ & \\
\hline $11 . \mathrm{S}^{\mathrm{d}}$ & 10:00:08.91 +02:40:10.6 & 10:00:08.94 +02:40:12.3 & $9.3 \pm 1.4$ & $8.3 \pm 1.8$ & $4.4 \pm 2.1$ & \\
\hline COSMOS23 & $10: 00: 10.12+02: 13: 34.6$ & Undetected & $8.4 \pm 0.9$ & $8.2 \pm 1.4$ & $<9.3$ & \\
\hline AzTEC6 $^{c}$ & $10: 00: 06.64+02: 38: 34.6$ & $10: 00: 06.50+02: 38: 37.7$ & $8.9 \pm 1.4$ & $8.0 \pm 1.8$ & $8.6 \pm 1.3$ & \\
\hline $\mathrm{AzTEC}^{\mathrm{c}}$ & $09: 59: 31.68+02: 30: 42.5$ & $09: 59: 31.72+02: 30: 44.0$ & $9.3 \pm 1.5$ & $7.9 \pm 1.9$ & $14.4 \pm 1.9$ & \\
\hline COSMOS22 & $09: 59: 33.55+02: 23: 46.5$ & $09: 59: 33.55+02: 23: 46.5$ & $8.5 \pm 1.2$ & $7.8 \pm 1.6$ & $8.9 \pm 2.2$ & $<8.9$ \\
\hline COSMOS24 & $09: 59: 12.08+02: 09: 54.5$ & $09: 59: 12.17+02: 09: 57.1$ & $7.9 \pm 1.1$ & $7.2 \pm 1.3$ & $8.1 \pm 1.7$ & $7.0_{-1.6}^{+1.6}$ \\
\hline COSMOS25 & $10: 00: 23.73+02: 19: 14.6$ & Undetected & $7.2 \pm 1.0$ & $7.1 \pm 1.1$ & $<9.3$ & \\
\hline COSMOSO1 $^{\mathrm{f}}$ & $10: 02: 09.77+02: 36: 33.9$ & $10: 02: 09.64+02: 36: 32.5$ & $20.3 \pm 3.3$ & & $10.6 \pm 1.2$ & \\
\hline $\operatorname{COSMOS} 02^{\mathrm{f}}$ & $10: 02: 49.22+02: 32: 55.1$ & $10: 02: 49.19+02: 32: 55.3$ & $20.2 \pm 3.6$ & & $18.6 \pm 0.7$ & \\
\hline
\end{tabular}

c From Younger et al. (2007) using the SMA at $890 \mu \mathrm{m}$, following the naming convention in their paper.

${ }^{\mathrm{d}}$ From Younger et al. (2009) using the SMA at $890 \mu \mathrm{m}$, following the naming convention in their paper.

e Also detected with PdBI in Smolčić et al. (2012b).

${ }^{\mathrm{f}}$ Source is found in the CLS maps but outside the area defining the CLS catalogue, and hence not used in our analysis. 
Table A4. SMA sample for the LHN field, ordered by decreasing deboosted SCUBA-2 flux density. All observations are from this work. Values of N/A in the $S_{\mathrm{SMA}}$ column indicate sources where our deboosting simulation was not applicable.

\begin{tabular}{|c|c|c|c|c|c|c|}
\hline Source & $\begin{array}{c}\text { RA/Dec SCUBA-2 } \\
(\mathrm{J} 2000)\end{array}$ & $\begin{array}{l}\text { RA/Dec SMA } \\
\quad(\mathrm{J} 2000)\end{array}$ & $S_{\mathrm{S} 2}^{\mathrm{obs}}[\mathrm{mJy}]$ & $S_{\mathrm{S} 2}[\mathrm{mJy}]$ & $S_{\mathrm{SMA}}^{\mathrm{obs}}[\mathrm{mJy}]$ & $S_{\mathrm{SMA}}[\mathrm{mJy}]$ \\
\hline LHN01 & $10: 46: 45.01+59: 15: 39.8$ & $10: 46: 45.00+59: 15: 41.6$ & $12.3 \pm 1.2$ & $12.3 \pm 1.8$ & $10.3 \pm 1.9$ & $8.8_{-1.7}^{+1.8}$ \\
\hline LHN02 & $10: 46: 35.78+59: 07: 48.0$ & $10: 46: 35.91+59: 07: 48.1$ & $12.0 \pm 1.0$ & $11.9 \pm 1.2$ & $12.2 \pm 1.9$ & $10.4_{-1.7}^{+2.0}$ \\
\hline LHN03a & $10: 47: 27.66+58: 52: 14.6$ & $10: 47: 27.97+58: 52: 14.1$ & $10.4 \pm 1.1$ & $9.9 \pm 1.3$ & $8.1 \pm 1.8$ & $7.3_{-1.8}^{+1.5}$ \\
\hline $03 b$ & $10: 47: 27.66+58: 52: 14.6$ & $10: 47: 26.52+58: 52: 12.8$ & $10.4 \pm 1.1$ & $9.9 \pm 1.3$ & $8.0 \pm 1.9$ & $\begin{array}{l}7.1_{-1.9}^{+1.8} \\
\end{array}$ \\
\hline LHN06 & $10: 45: 55.19+59: 15: 28.1$ & $10: 45: 55.24+59: 15: 28.6$ & $9.7 \pm 1.1$ & $9.7 \pm 0.9$ & $7.2 \pm 1.8$ & $6.6_{-65}^{+1.5}$ \\
\hline LHN04 & $10: 48: 03.37+58: 54: 22.9$ & $10: 48: 03.57+58: 54: 21.5$ & $10.1 \pm 1.3$ & $8.9 \pm 1.4$ & $14.1 \pm 2.4$ & $11.7_{-2.5}^{+2.2}$ \\
\hline LHN08 & $10: 47: 00.03+59: 01: 07.5$ & $10: 47: 00.18+59: 01: 07.5$ & $9.2 \pm 1.0$ & $8.9 \pm 1.6$ & $10.4 \pm 1.6$ & $9.4_{-1.6}^{+1.4}$ \\
\hline LHN11 & $10: 45: 22.55+59: 17: 21.7$ & Undetected & $8.6 \pm 1.4$ & $8.8 \pm 1.7$ & $<7.21 .8$ & \\
\hline LHN07 & $10: 45: 35.23+58: 50: 49.9$ & $10: 45: 34.98+58: 50: 49.9$ & $9.3 \pm 1.1$ & $8.7 \pm 1.4$ & $9.6 \pm 1.6$ & $8.8_{-16}^{+1.3}$ \\
\hline LHN10 & $10: 45: 54.58+58: 47: 54.1$ & $10: 45: 54.50+58: 47: 55.6$ & $8.8 \pm 1.1$ & $8.3 \pm 1.5$ & $8.2 \pm 0.8$ & $8.1_{-0.8}^{-1.6}$ \\
\hline LHN05 & $10: 43: 51.48+59: 00: 57.7$ & $10: 43: 51.21+59: 00: 58.1$ & $10.0 \pm 1.5$ & $8.2 \pm 2.1$ & $10.9 \pm 2.4$ & $8.8_{-2.3}^{+0.0}$ \\
\hline LHN09 & $10: 45: 23.87+59: 16: 25.7$ & $10: 45: 23.11+59: 16: 18.6$ & $9.0 \pm 1.3$ & $8.2 \pm 1.5$ & $9.4 \pm 1.5$ & $8.6_{-1.4}^{+1.3}$ \\
\hline LHN12 & $10: 46: 32.85+59: 02: 12.0$ & Undetected & $8.6 \pm 1.0$ & $8.1 \pm 1.3$ & $<8.0$ & \\
\hline LHN13a & $10: 47: 25.25+59: 03: 40.7$ & $10: 47: 25.47+59: 03: 36.7$ & $8.5 \pm 1.1$ & $7.9 \pm 1.4$ & $5.5 \pm 0.8$ & $\mathrm{~N} / \mathrm{A}$ \\
\hline $13 b$ & $10: 47: 25.25+59: 03: 40.7$ & $10: 47: 25.13+59: 03: 41.5$ & $8.5 \pm 1.1$ & $7.9 \pm 1.4$ & $3.9 \pm 0.8$ & $\mathrm{~N} / \mathrm{A}$ \\
\hline LHN14 & $10: 46: 31.68+58: 50: 54.0$ & $10: 46: 31.58+58: 50: 55.7$ & $8.5 \pm 1.1$ & $7.9 \pm 1.4$ & $7.1 \pm 0.8$ & $7.0_{-0.8}^{+0.7}$ \\
\hline LHN15 & $10: 46: 57.26+59: 14: 57.6$ & $10: 46: 57.30+59: 14: 58.6$ & $8.5 \pm 1.2$ & $7.9 \pm 0.9$ & $5.5 \pm 0.7$ & $5.5_{-0.8}^{+0.6}$ \\
\hline LHN16 & $10: 44: 56.86+58: 49: 59.0$ & $10: 44: 56.74+58: 49: 59.7$ & $8.3 \pm 1.1$ & $7.6 \pm 1.4$ & $16.9 \pm 2.5$ & $13.9_{-2.2}^{+3.0}$ \\
\hline LHN17 & $10: 44: 47.69+59: 00: 36.6$ & $10: 44: 47.68+59: 00: 35.6$ & $8.1 \pm 1.1$ & $7.5 \pm 1.3$ & $5.6 \pm 0.7$ & $5.5_{-0.7}^{+0.7}$ \\
\hline LHN18 & $10: 47: 20.57+59: 10: 40.9$ & $10: 47: 20.54+59: 10: 43.4$ & $8.1 \pm 1.1$ & $7.3 \pm 1.3$ & $7.0 \pm 0.8$ & $6.9_{-0.7}^{+0.7}$ \\
\hline
\end{tabular}

Table A5. SMA sample for the EGS field, ordered by decreasing deboosted SCUBA-2 flux density. All observations are from this work.

\begin{tabular}{lccrrrr}
\hline Source & $\begin{array}{c}\text { RA/Dec SCUBA-2 } \\
(\mathrm{J} 2000)\end{array}$ & $\begin{array}{c}\text { RA/Dec SMA } \\
(\mathrm{J} 2000)\end{array}$ & \multicolumn{1}{c}{$S_{\mathrm{S} 2}^{\text {obs }}[\mathrm{mJy}]$} & $S_{\mathrm{S} 2}[\mathrm{mJy}]$ & $S_{\text {SMA }}^{\text {obs }}[\mathrm{mJy}]$ & $S_{\mathrm{SMA}}[\mathrm{mJy}]$ \\
\hline EGS01 & $14: 19: 51.56+53: 00: 44.8$ & $14: 19: 51.33+53: 00: 46.4$ & $16.3 \pm 1.2$ & $16.3 \pm 1.4$ & $13.2 \pm 0.9$ & $12.9_{-0.8}^{+0.9}$ \\
EGS02 & $14: 15: 57.62+52: 07: 11.1$ & $14: 15: 57.53+52: 07: 12.7$ & $12.7 \pm 1.3$ & $12.1 \pm 1.2$ & $13.8 \pm 1.4$ & $12.9_{-1.2}^{+1.5}$ \\
EGS03 & $14: 15: 47.46+52: 13: 47.2$ & $14: 15: 47.09+52: 13: 48.6$ & $10.8 \pm 1.0$ & $10.5 \pm 1.1$ & $16.4 \pm 2.8$ & $12.9_{-2.4}^{+2.9}$ \\
EGS05 & $14: 19: 20.35+52: 56: 08.9$ & $14: 19: 20.08+52: 56: 09.1$ & $10.7 \pm 1.0$ & $10.1 \pm 1.4$ & $20.0 \pm 0.9$ & $19.7_{-0.8}^{+1.0}$ \\
EGS06 & $14: 17: 40.55+52: 29: 04.7$ & $14: 17: 40.34+52: 29: 06.7$ & $10.0 \pm 1.0$ & $9.8 \pm 2.3$ & $9.8 \pm 2.0$ & $8.9_{-1.8}^{+1.7}$ \\
EGS08 & $14: 19: 00.37+52: 49: 45.3$ & $14: 19: 00.24+52: 49: 48.3$ & $10.4 \pm 1.1$ & $9.8 \pm 1.5$ & $8.6 \pm 1.5$ & $8.1_{-1.4}^{+1.5}$ \\
EGS04 & $14: 19: 14.54+53: 00: 33.6$ & $14: 19: 14.32+53: 00: 33.8$ & $10.5 \pm 1.4$ & $9.3 \pm 1.6$ & $11.1 \pm 1.5$ & $10.5_{-1.6}^{+1.2}$ \\
EGS10 & $14: 17: 44.09+52: 21: 22.4$ & $14: 17: 43.38+52: 21: 21.7$ & $10.2 \pm 1.5$ & $9.2 \pm 2.3$ & $8.3 \pm 1.6$ & $7.7_{-1.5}^{+1.6}$ \\
EGS11 & $14: 17: 41.73+52: 22: 04.6$ & $14: 17: 41.41+52: 22: 07.9$ & $9.8 \pm 1.4$ & $9.2 \pm 1.4$ & $7.2 \pm 1.5$ & $6.7_{-1.3}^{+1.6}$ \\
\hline EGS07 $^{\mathrm{f}}$ & $14: 18: 22.09+52: 54: 01.0$ & $14: 18: 22.04+52: 54: 02.0$ & $10.6 \pm 1.6$ & & $7.7 \pm 1.5$ & \\
EGS09 $^{\mathrm{f}}$ & $14: 20: 52.38+52: 54: 02.0$ & $14: 20: 52.55+52: 54: 00.3$ & $10.5 \pm 1.6$ & & $6.1 \pm 1.4$ & \\
\hline
\end{tabular}

\footnotetext{
${ }^{\mathrm{f}}$ Source is found in the CLS maps but outside the area defining the CLS catalogue, and hence not used in our analysis.
} 